\title{
Mechanisms of Single-Walled Carbon Nanotube Nucleation, Growth and Chirality-Control: Insights from QM/MD Simulations
}

\author{
Alister J. Page ${ }^{1}$, Ying Wang2, K. R. S. Chandrakumar', \\ Stephan Irle 2 and Keiji Morokuma ${ }^{1,3}$ \\ ${ }^{1}$ Fukui Institute for Fundamental Chemistry, Kyoto University, \\ 2Institute for Advanced Research and Department of Chemistry, Nagoya University \\ ${ }^{3}$ Cherry L. Emerson Centre for Scientific Computation and Department of Chemistry, \\ Emory University, \\ 1,2Japan \\ ${ }^{3}$ U.S.A.
}

\section{Introduction}

The experimental characterisations of carbon nanotubes (CNTs) (Iijima, 1991) and in particular single-walled CNTs (SWNTs) (Iijima \& Ichihashi, 1993) in the early 1990s were landmark moments in $20^{\text {th }}$ century science. The potential uses of these remarkable nanostructures are now becoming realised, as their synthesis is now routinely performed on the industrial scale. The initial successes in this respect were generally experimental techniques that were previously well established in other fields. This is particularly true of the chemical vapor deposition (CVD) and arc-discharge processes. The original experimental characterisation of SWNTs was in fact accomplished using nanotubes synthesised with the former method (Iijima \& Ichihashi, 1993). The understanding of the way in which CNTs nucleate and grow was therefore synergic with the evolution and refinement of these synthetic methods. Indeed, the original mechanisms of CNT nucleation and growth were conceived from experimental observations. The most prevalent of these today is the vapor-liquid-solid (VLS) mechanism (Saito, 1995). According to this mechanism, SWNT nucleation growth is postulated to consist of three distinct stages. The first of these features a mixed carbon/catalyst vapor phase, from which co-condensation yields liquid catalyst-carbide nanoparticles. Typical catalysts in the growth of SWNTs are traditionally transition metals such as $\mathrm{Fe}, \mathrm{Ni}, \mathrm{Co}$, Mo, and alloys thereof (see (Journet et al., 1997; Moisala et al., 2003; Harris, 2007), and references therein). The precipitation of atomic carbon from this liquid carbide phase takes place once the carbide phase is saturated with carbon. This precipitation yields the formation of solid phase CNTs. Due to the inherent limits in spatial and temporal resolutions that are furnished by experimental techniques and instrumentation, there inevitably remain questions regarding the VLS mechanism and CNT growth that, for now, cannot be answered from an experimental standpoint. There are several infamous examples in this respect. For instance, the mechanism of so-called 'catalyst-free' SWNT nucleation growth remains unknown, following the recent 
experimental reports demonstrating the growth of CNTs in the absence of a transition metal catalyst. The factors that govern 'chirality-controlled' growth (i.e. growth that produces a specific $(n, m)$ chiral SWNT, as opposed to a broad distribution of $(n, m)$ SWNTs) have also remained elusive to date.

It is in this respect that theoretical models of CNT growth have recently come to the fore by complementing, and in some cases pre-empting, experimental understanding of SWNT nucleation and growth. The most notable theoretical approach in this respect is molecular dynamics (MD). In this work, we will highlight the recent advances made in our understanding of SWNT nucleation and growth mechanisms gained from quantum mechanical MD (QM/MD) simulations. Following a brief review of experimental SWNT synthesis (\$1.1) and previous theoretical investigations of SWNT nucleation and growth (\$1.2), we will briefly outline the quantum chemical approach used in our simulations (\$2). A discussion of QM/MD simulations of SWNT nucleation on both Fe and Ni catalysts will be presented in $\S 3$. SWNT nucleation as the result of gas-phase acetylene, $\mathrm{C}$ and $\mathrm{C}_{2}$ adsorption, as well as the decomposition of the $\mathrm{Fe}$ - and Ni-carbide phases will be considered here. More recent simulations concerning the mechanism of SWNT nucleation on Si-based catalysts, particularly $\mathrm{SiO}_{2}, \mathrm{SiC}$ and $\mathrm{Si}$, will then be discussed in $\$ 3.4$. In §4 we will discuss $\mathrm{QM} / \mathrm{MD}$ simulations concerning the continued growth of SWNTs on Fe and Ni catalysts. Finally, insights gained from recent $\mathrm{QM} / \mathrm{MD}$ simulations regarding the issue of chiralitycontrolled growth will be the subject of $\$ 5$.

\subsection{Experimental synthesis of SWNTs}

An exhaustive review of experimental techniques of CNT and SWNT synthesis lies beyond the scope of the current work. Instead, we will provide a cursory overview of two relevant experimental techniques pertinent to the simulations presented in this work, viz. the CVD and arc-discharge techniques on transition metal catalysts. We will also briefly summarise recent experimental reports concerning the synthesis of CNTs on 'non-traditional' catalysts such as $\mathrm{SiO}_{2}, \mathrm{SiC}$ and $\mathrm{Al}_{2} \mathrm{O}_{3}$. For more extensive reviews of this area, we direct the reader to the several books (Dresselhaus et al., 1996; Dresselhaus et al., 2001) and reviews (Teo et al., 2004; Yoshinori, 2004) that are concerned with experimental synthesis.

The CVD process is widely used throughout the areas of material science and solid-state physics. Put simply, it involves a substrate material being deposited and subsequently exposed to a chemical reagent. This reagent subsequently decomposes or reacts on the substrate surface, yielding a desired chemical deposit. In the context of SWNT synthesis, the substrate is traditionally a thin (typically $<100 \mathrm{~nm}$ ) layer of transition metal nanoparticles, and the reagent is a carbonaceous gas such as methane, acetylene or ethanol. These gaseous reagents are believed to decompose on the catalyst nanoparticle surface, thus providing a source of atomic carbon, before dissolving and diffusing into the metal catalyst. As will be shown in $\$ 3$, however, there is currently a lack of consensus over exactly how these initial stages of SWNT nucleation occur. The metal catalyst layer itself may be deposited via a number of different techniques. However, SWNT synthesis is only successful with relatively small nanoparticle diameters (ca. $5 \mathrm{~nm}$ or less) (Teo et al., 2004). The catalytic species that are typically used for the synthesis, or growth, of SWNTs include first row transition metals such as Fe, Ni, Co and Mo (Journet et al., 1997; Moisala et al., 2003; Harris, 2007). These metals, and alloys thereof, are employed today in the industrial scale CVD synthesis of SWNTs. However, the alkaline earth metals $\mathrm{Mg}$ and $\mathrm{Ca}$, as well as $\mathrm{Ir}$ and $\mathrm{W}$ have also shown catalytic capabilities in the context of CVD SWNT synthesis (Esconjauregui et al., 2009). Most recently, so-called 'catalyst-free' synthesis of SWNTs has been reported (see 
(Homma et al., 2009), and references therein). In these cases, SWNTs have been synthesised via CVD in the absence of the metal catalyst layer. It follows then that the cracking/decomposition of the gaseous reagent takes place on the supporting substrate itself. The precise mechanism underpinning this un-catalysed reagent decomposition is as yet not unknown. While a number of such covalent CVD 'catalysts' have been reported (including $\mathrm{Ge}$ (Takagi et al., 2007), $\mathrm{Al}_{2} \mathrm{O}_{3}$ (Liu et al., 2008; Liu et al., 2010a) and even other nanocarbon structures (Homma et al., 2009)) the majority of experimental investigation in this area has so far focused upon $\mathrm{SiO}_{2}$ (Bachmatiuk et al., 2009; Huang et al., 2009; Liu et al., 2009a; Liu et al., 2009b; Liu et al., 2010b).

In the most general terms, the arc-discharge method involves an electrical discharge between two electrodes through a particular gas (in the process of which this gas is broken down). This method was employed in the synthesis of $\mathrm{C}_{60}$ fullerene (Krätschmer et al., 1990; Heath, 1992), before becoming a popular synthetic method for SWNTs. Indeed, the first experimental characterisation of SWNTs (Iijima \& Ichihashi, 1993) employed arc-discharge synthesis. In both fullerene and CNT synthesis, the arc-discharge method involves the electrical discharge between two carbon electrodes (one of which is vaporised), usually in the presence of some inert buffer/carrier gas. In the case of SWNT synthesis however, it is preferable that a metal-doped anode is used, otherwise multi-walled carbon nanotubes (MWNTs) are formed. Thus, both the carbon and metal vapors may co-condense, forming liquid phase metal carbide particles. In this sense, the arc-discharge technique constitutes a 'pure' example of the VLS mechanism of SWNT growth. The most common catalytic metals employed in this arc-discharge SWNT synthesis are the same as those employed in the traditional CVD synthesis of SWNTs (i.e. Fe, Co, Mo etc.) (Journet \& Bernier, 1998). There is great variability, however, regarding the yield and diameter distribution and of the synthesised nanotubes with different catalyst metals. The same may be said regarding the pressure and composition of the environmental buffer gas (Journet \& Bernier, 1998; Farhat et al., 2001).

\subsection{Theoretical investigations of SWNT growth}

The first foray of theoretical investigation aimed at understanding CNT nucleation and growth took place in the 1990s (see (Irle et al., 2009), and references therein). Amongst the first of these undertakings was that of Smalley et al., who demonstrated that a single metal atom ( $\mathrm{Ni}$ or $\mathrm{Co}$ ) has the ability to prevent the closure of an extended $s p^{2}$-hybridised carbon structure, simply by 'scooting' around the open edge (Thess et al., 1996). Despite the simplicity of this 'scooter mechanism', it revealed for the first time this most fundamental property that is now commonly ascribed to the catalytic nanoparticle during SWNT growth. Another early discovery made by Smalley et al. concerned the observation of SWNT growth during the Boudouard reaction $\left(2 \mathrm{CO} \rightarrow \mathrm{C}+\mathrm{CO}_{2}\right)$ on nanoparticle Mo catalysts (Dai et al., 1996). It was in this investigation that the now well-known 'yarmulke' mechanism was proposed. This mechanism postulates that a SWNT 'cap' fragment exists on the catalyst nanoparticle surface prior to the continued growth of the nanotube, a fact that has since been corroborated independently on a number of occasions (see (Irle et al., 2009), and references therein).

It was not until 2002 that MD simulations were employed successfully in simulating the SWNT nucleation and growth processes. In a series of investigations, Shibuta and coworkers simulated Ni-catalysed SWNT nucleation (Shibuta \& Maruyama, 2002; 2003). The roles of both $\mathrm{Ni}$ vapor and condensed $\mathrm{Ni}$ nanoparticles in the earliest stages of the SWNT nucleation process were therefore established. These theoretical investigations proved 
remarkably consistent with contemporary experimental work. Moreover, the previous prediction made by Smalley et al. regarding the catalyst preventing closure of the nascent SWNT was realized in situ. In a somewhat different approach, Bolton and co-workers investigated the relationship between diffusion and precipitation of carbon from $\mathrm{Fe}$ nanoparticles, and SWNT nucleation in a series of investigations (Ding et al., 2004a; Ding et al., 2004c; b; Ding et al., 2006a; Ding et al., 2006b). Notably, the nucleation of SWNTs from Fe 'carbide' was demonstrated at temperatures between 800 and $1400 \mathrm{~K}$ (Ding et al., 2004a). According to this investigation, the formation of the SWNT cap structure was preceded by three distinct stages: (1) the incorporation of carbon into the Fe nanoparticle bulk; (2) the saturation of the Fe nanoparticle with atomic carbon, and (3) the formation of polyyne chains and small graphitic 'islands' on the Fe nanoparticle surface. It was also noted that at lower temperatures (below $600 \mathrm{~K}$ ), encapsulation of the nanoparticle, rather than the formation of a well-defined cap structure, took place. These efforts were complemented by investigations in 2007 (Shibuta \& Maruyama, 2007a; b) in which an inverse relationship between the melting behaviors of catalyst nanoparticles and the carbon-catalyst interaction was observed. This was in agreement with an earlier independent investigation (Ding et al., $2006 \mathrm{~b})$. These authors have also investigated the relative behaviors of $\mathrm{Fe}-, \mathrm{Ni}-$ and $\mathrm{Co}-$ carbide nanoparticles (Shibuta \& Maruyama, 2007b), and observed that the Co-C interaction exceeds both the $\mathrm{Fe}-\mathrm{C}$ and $\mathrm{Ni}-\mathrm{C}$ interactions. This correlation suggests that a stronger catalyst-carbon interaction may yield more defective synthetic SWNTs.

The pioneering MD investigations in this area relied on the reactive empirical bond order (REBO) force field (Brenner, 1990; 1992; Brenner et al., 2002), which is itself based upon the Tersoff interactive potential (Tersoff, 1988; 1989). While the use of this force-field makes MD simulations on nanosecond timescales possible, it nonetheless has several notable deficiencies with respect to the chemistry of SWNT nucleation. For instance, $\pi$-conjugation and aromatic stabilization of carbon (central to the formation and extension of an $s p^{2}$ hybridised carbon network, such as a CNT), charge transfer effects and the near-degeneracy of transition metal $d$-orbitals (crucial in the case of transition metal catalysts) can not be accurately described by the REBO or Tersoff potentials. One infamous outcome of the former of these deficiencies is the overestimation and underestimation of the $s p^{3}$ - and $s p$ hybridised carbon fractions, respectively, during the self-assembly of fullerenes at high temperature (Zheng et al., 2004; Irle et al., 2006). On a few occasions (Gavillet et al., 2001; Raty et al., 2005), more reliable simulations based on Carr-Parinello MD (CPMD) have been reported. The description of atomic interaction and bonding in these latter investigations relied on density functional theory (DFT), and so therefore significantly exceeded that given by the REBO potential. However, this advantage incurs a substantial increase in the computational cost of the calculation. Thus, these simulations employed generally unphysical assumptions, or model systems, in order to alleviate these computational costs. In addition, the timescales of these simulations were restricted to less than $25 \mathrm{ps}$, and as such can hardly be considered to be sufficient in the context of SWNT growth. While these simulations nevertheless represent Herculean efforts, relatively few conclusions regarding the mechanisms of SWNT nucleation and growth have been gained as a result. Ideally, the most suitable approach to the simulation of SWNT nucleation and growth would provide a compromise between quantum mechanical accuracy, and the computational efficiency provided by semi-empirical, or classical, force-field based methods. In the subsequent section, we will turn to one such method, the density-functional tight-binding (DFTB) method, and provide a brief picture of its formulation. 


\section{QM/MD method: Theoretical background}

In this section we will present a brief account of the main theoretical methods and algorithms employed in the QM/MD simulations that are presented in \$3-5. Our approach is based on the DFTB method. In essence this method is a two-centre approximation to the popular DFT method, which has its origins in the 1990s (Porezag et al., 1995; Seifert et al., 1996). Consequently, for systems consisting of hundreds of atoms (such as those considered here), DFTB is $c a$. three orders of magnitude faster than traditional DFT methods. In DFTB theory, the atomic/molecular energy is given as,

$$
E_{\mathrm{DFTB}}=\sum_{i}^{\text {occ. }} \varepsilon_{\mathrm{i}}+\frac{1}{2} \sum_{A \neq B}^{\text {atoms }} E_{\mathrm{rep}}^{A B}
$$

where $\varepsilon_{\mathrm{i}}$ is the $i^{\text {th }}$ Kohn-Sham eigenvalue (obtained from the diagonalization of the Hamiltonian matrix in the atomic orbital basis), and $E_{\text {rep }}^{A B}$ describes the repulsive force between nuclei $A$ and $B$. It is noted that the Hamiltonian matrix elements from which the $\varepsilon_{\mathrm{i}}$ 's are computed via diagonalization need only be computed once (i.e. during the development of a particular set of DFTB parameters). The Hamiltonian matrix elements and $E_{\text {rep }}^{A B}$ potentials are subsequently stored in memory and recalled for each subsequent DFTB calculation. This results in a significant reduction in the computation time compared to traditional DFT. Since DFTB is based upon DFT, it inherits both the strengths and weaknesses of DFT. Molecular geometries and vibration frequencies calculated using DFTB are therefore generally reliable. On the other hand, DFTB poorly describes systems exhibiting dispersive or multi-reference characters. The description of conduction bands etc. is also limited with DFTB, as it is a minimal basis set method. In addition, although the standard DFTB method describes homonuclear and ionic systems very well, it is unable to describe accurately systems exhibiting a degree of charge transfer. To this end, the selfconsistent-charge DFTB (SCC-DFTB) method was developed (Elstner et al., 1998). The SCCDFTB energy is given as,

$$
E_{\text {SCC-DFTB }}=\sum_{i}^{\text {occ. }} \varepsilon_{\mathrm{i}}+\frac{1}{2} \sum_{A \neq B}^{\text {atoms }} E_{\text {rep }}^{A B}+\frac{1}{2} \sum_{A \neq B}^{\text {atoms }} \gamma_{A B} \Delta q_{A} \Delta q_{B}
$$

The SCC-DFTB energy includes a 2nd order contribution to the DFTB energy involving the charge fluctuation, $\Delta q_{\alpha}=q_{\alpha}-q_{\alpha}^{0}$, where $q_{\alpha}$ and $q_{\alpha}^{0}$ are the molecular and lone-atom nuclear charges, respectively. The SCC-DFTB molecular orbitals (MOs) are iteratively optimized until the corresponding energy of equation (2) becomes self-consistent with respect to $\Delta q_{A}$ and $\Delta q_{B}$. Typically this iterative solution incurs an increase in computational time of approximately one order of magnitude with respect to DFTB. In these cases it is common that self-consistency with respect to $\Delta q_{A}$ and $\Delta q_{B}$ cannot be attained. However, this convergence issue is improved dramatically by introducing a finite electronic temperature during the convergence of the MOs. In such a case, the variational SCC-DFTB energy becomes,

$$
E_{\text {SCC-DFTB }}+T_{e} 2 k_{B} \sum_{i}^{\text {orbitals }}\left[f_{i} \ln f_{i}+\left(1-f_{i}\right) \ln \left(1-f_{i}\right)\right]
$$


where $T_{e}$ is the electronic temperature, and the population of the $i^{\text {th }} \mathrm{MO}$ is now defined using the Fermi-Dirac distribution,

$$
f_{i}=\frac{1}{\exp \left[\left(\varepsilon_{i}-\mu\right) / k_{B} T_{e}+1\right]}
$$

Note that this occupation is a continuous function of the $i$ th $\mathrm{MO}$ energy, $\varepsilon_{i}$, and $\mu$ is the chemical potential. This function is continuous over $[0,1]$ (and typically varies near the Fermi level).

The MD method essentially involves the discrete integration of Newton's equations of motion as a function of time. Since its conception (Alder \& Wainwright, 1957; Rahman, 1964), it has been applied with great success in fields as diverse as molecular physics, materials science and biological sciences. The discretization of time in MD integration may be achieved in a number of different ways. One such method is the Velocity-Verlet algorithm (Swope et al., 1982), which is perhaps the most popular MD integration scheme today. In this algorithm both the nuclear coordinates and velocities are updated at each iteration of the integration, using coordinates/velocities of the previous iteration,

$$
\begin{gathered}
\mathbf{x}(t+\Delta t) \approx \mathbf{x}(t)+\mathbf{v}(t) \Delta t-\frac{1}{2 \mathbf{m}} \nabla U(\mathbf{x}(t)) \Delta t^{2} \\
\mathbf{v}(t+\Delta t) \approx \mathbf{v}(t)-\frac{1}{2 \mathbf{m}}[\nabla U(\mathbf{x}(t))+\nabla U(\mathbf{x}(t+\Delta t))] \Delta t
\end{gathered}
$$

where $U$ is the derivative of the electronic potential energy (in this case calculated using DFTB). Discrete integration of the equations of motion in this fashion results in the micro-canonical, or NVE, ensemble (in which the number of atoms, $N$, the volume, $V$, and the total energy, $E$, of the system are held constant). We will limit the present discussion to MD in which $N, V$, and the system temperature, $T$, are held constant throughout the simulation. Placing these restrictions on the MD system results in what is otherwise known as the NVT ensemble. There are several popular methods (more commonly known as thermostats) by which the MD temperature is maintained, and each results in the re-scaling of nuclear velocities in some way. Of particular note are the thermostats of Anderson (Andersen, 1980), Berendsen (Berendsen et al., 1984) and the method of velocity scaling (Woodcock, 1971). In the present context, we employ the NoséHoover chain thermostat (Nose, 1984; Hoover, 1985; Martyna et al., 1992; Martyna et al., 1996), in which the Hamiltonian of the system is augmented with a term representing a heat-bath that is coupled to the degrees of freedom of the system. The augmented equations of motion thus sample microcanonical and canonical distributions in the extended and original systems, respectively. However, care must be taken when deciding the strength at which the NoseHoover chain thermostat is coupled to the MD system. Coupling that is too weak will result in inadequate temperature control, whereas coupling that is too strong is known to result in highfrequency temperature oscillations, and consequently unreliable dynamics.

\section{QM/MD simulations of SWNT nucleation}

We turn now to a discussion of recent QM/MD simulations of SWNT nucleation. This discussion will focus on the mechanism of SWNT nucleation on a number of different 
catalyst nanoparticles, including $\mathrm{Fe}, \mathrm{Ni}, \mathrm{SiO}_{2}, \mathrm{SiC}$ and $\mathrm{Si}$. SWNT nucleation resulting from both model CVD and arc-discharge processes will also be discussed. In this way we will demonstrate that, at the atomic level, the mechanism of SWNT nucleation is surprisingly invariant to both the experimental method employed, and several pertinent environmental factors. We begin with the case of CVD on Fe catalyst nanoparticles.

\subsection{Acetylene CVD and SWNT nucleation on Fe catalysts}

Today, CVD synthesis of SWNTs is perhaps the most popular method of choice on the commercial scale. The most typically employed gaseous precursors include acetylene, ethanol and methane (almost always in the presence of some inert buffer/carrier gas). Yet little was known regarding the atomistic mechanism of such carbonaceous CVD processes until relatively recently. Such knowledge was furnished entirely by theoretical MD simulations, and in particular QM/MD simulations. Here we will focus on the mechanism of Fe-catalysed acetylene CVD elucidated from such recent QM/MD simulations.

In order to investigate the Fe-catalysed acetylene $\left(\mathrm{C}_{2} \mathrm{H}_{2}\right)$ CVD process, we employed an $\mathrm{Fe}_{38}$ catalyst nanoparticle. The diameter of this nanoparticle is $c a .0 .7 \mathrm{~nm}$, and so is of comparable diameter to experimental SWNT diameter distributions (Sugai et al., 2003). Gas-phase $\mathrm{C}_{2} \mathrm{H}_{2}$ molecules were initially adsorbed onto the equilibrated catalyst nanoparticle (see Fig. 1a), after which the resultant $30 \mathrm{C}_{2} \mathrm{H}_{2}-\mathrm{Fe}_{38}$ model complex was relaxed at $1500 \mathrm{~K}$ for 500 ps. During the $\mathrm{C}_{2} \mathrm{H}_{2}$ adsorption process the occasional abstraction of atomic $\mathrm{H}$ by the Fe catalyst surface was observed, thus forming $\mathrm{C}_{2} \mathrm{H}$ radicals. Similarly, abstraction of atomic $\mathrm{H}$ by adjacent $\mathrm{C}_{2} \mathrm{H}_{2}$ molecules was also observed, resulting in both $\mathrm{C}_{2} \mathrm{H}$ and $\mathrm{C}_{2} \mathrm{H}_{3}$ moieties. Both abstraction processes are endothermic, with barriers between $\mathrm{ca} .20-35 \mathrm{kcal} \mathrm{mol}^{-1}$. The direct formation of $\mathrm{H}_{2}$ was however not observed, despite the abstraction of atomic $\mathrm{H}$ by the catalyst surface. This is not surprising, considering the high endothermicity of the $\mathrm{H}_{2}$ formation process (using SCCDFTB, this barrier is estimated to be $c a .35-50 \mathrm{kcalmol}^{-1}$ ). Such endothermic processes are inherently difficult to observe in MD simulations on this time scale. The radical products $\mathrm{C}_{2} \mathrm{H}$ and $\mathrm{C}_{2} \mathrm{H}_{3}$ are extremely reactive, and therefore rapidly initiated oligomerisation between adjacent $\mathrm{C}_{2} \mathrm{H}_{x}$ species. Such oligomerisation is exothermic by ca. $18 \mathrm{kcal} \mathrm{mol}^{-1}$ (see Fig. 1b). Following these oligomerisation reactions, extended $s p^{2}$-hybridised carbon networks ultimately form on the catalyst surface. The cross-linking of neighboring polyyne chains drove this process, and resulted in the formation of pentagonal and hexagonal carbon rings. In all cases, pentagonal rings were formed first - an observation that will frequently recur in \$3.23.4. Such a cross-linking process is depicted schematically in Fig. 2. Also depicted in Fig. 2 is the polyyne cross-linking mechanism (pertaining to SWNT growth) proposed by Eres (Eres et al., 2009). While both processes are distinctly similar, no hexagonal rings were formed in the cross-linking process in the present work.

Fig. 3 shows the ultimate product of this $\mathrm{H}$-abstraction and polyyne oligomerisation/crosslinking process, viz. the formation of an extended $s p^{2}$-hybridised carbon network. The structure of this network generally fell into one of three categories. The most 'successful' structure regarding SWNT nucleation is structure (i), in which the network extends over the catalyst surface. In essence such a structure constitutes a SWNT cap-fragment, similar to the 'yarmulke' cap proposed by Smalley and co-workers (Dai et al., 1996). However, structure (i) was only observed at relatively low $\mathrm{H} / \mathrm{C}$ ratios (see \$3.2-3.3 for examples of SWNT nucleation in the absence of $\mathrm{H}$ ). In cases of higher $\mathrm{H} / \mathrm{C}$ ratios (i.e. more $\mathrm{H}$ ), structure (ii) was typically observed, due to the passivative effect of $\mathrm{H}$ at the unsaturated edge of the carbon network. It is assumed that this graphene-like sheet may coalesce to form an open nanotube 
a

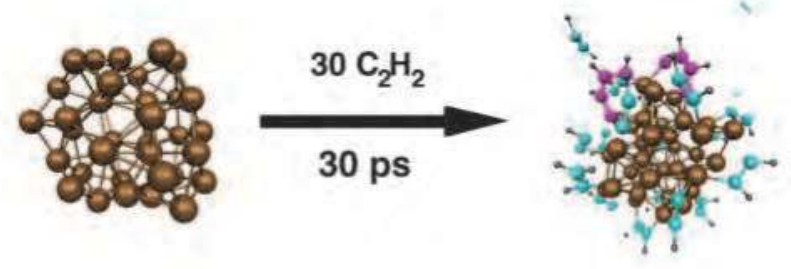

b
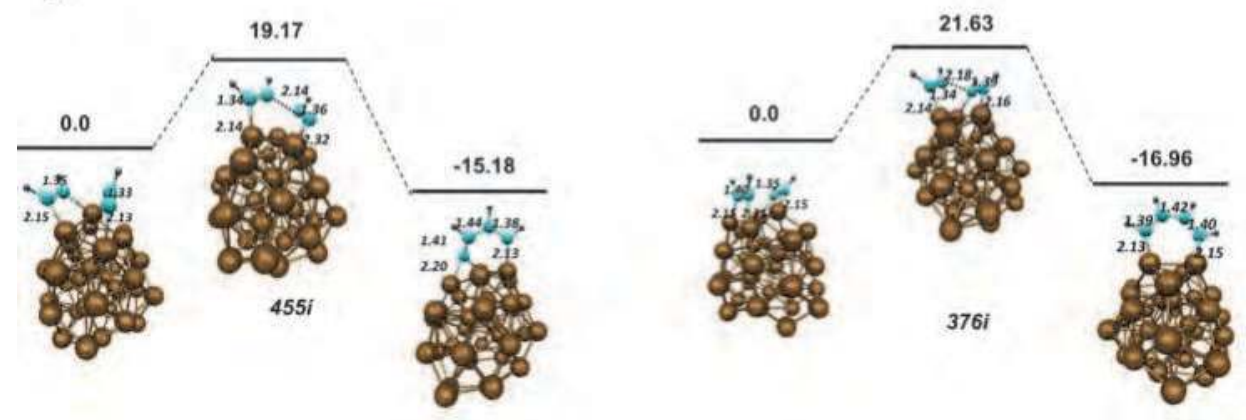

Fig. 1. The initial stages of simulated acetylene CVD on $\mathrm{Fe}_{38}$ catalyst nanoparticles at $1500 \mathrm{~K}$. a) Oligomerisation results in the formation of extended polyyne chains on the catalyst surface within $30 \mathrm{ps}$. Brown, cyan and grey spheres represent $\mathrm{Fe}, \mathrm{C}$ and $\mathrm{H}$ atoms, respectively. $s p^{2}$-hybridised $C$ atoms are represented by magenta spheres. b) Examples of $C_{2}$ oligomerisation, and their associated energetics. Examples of both the $\mathrm{C}_{2} \mathrm{H}_{2}+\mathrm{C}_{2} \mathrm{H} \rightarrow \mathrm{C}_{4} \mathrm{H}_{3}$ and $\mathrm{C}_{2} \mathrm{H}_{2}+\mathrm{C}_{2} \mathrm{H}_{2} \rightarrow \mathrm{C}_{4} \mathrm{H}_{4}$ oligomerisation reactions are depicted. The $\mathrm{C}_{2} \mathrm{H}$ precursors here are occasionally produced via the abstraction of atomic $\mathrm{H}$ onto the catalyst surface. All energies and bond lengths given in $\mathrm{kcalmol}^{-1}$ and $\AA$, respectively. Transition state imaginary frequencies are given in $\mathrm{cm}^{-1}$.

according to the mechanism proposed by Eres (Eres et al., 2009) (see Fig. 2). Structure (iii) was also observed as a result of the polyyne oligomerisation/cross-linking process. In this case, the process yielded 'islands' of $s p^{2}$-hybridised carbon. Assumedly, QM/MD relaxation of such structures over longer time scales (i.e. several nanoseconds) would yield a more consistent network, such as that typical of structure (i). Thus, it is demonstrated here that SWNT nucleation is not necessarily preceded by a carbon cap-structure, or a liquid carbide phase. The latter conclusion will be corroborated in \$3.2.

\subsection{SWNT nucleation on Fe \& Ni catalysts via adsorption of gas-phase $\mathrm{C}_{2}$}

It was observed in $\$ 3.1$ that the removal/sequestration of hydrogen from feedstock acetylene molecules was the most problematic issue in these QM/MD simulations. Presumably the same problem would exist regardless of the type of carbonaceous precursor employed in this respect (be it acetylene, methane, ethanol, etc.). This difficulty arises due to a problem inherent to MD methods, since such methods have difficulty overcoming large energy barriers on the global potential energy surface (PES). Although, in the limit of infinite time, an MD simulation will sample all possible geometrical configurations, and consequently will have overcome all such barriers on the global PES, 
in practice, such sampling is obviously not possible. To this end, we will discuss an alternative QM/MD approach to the problem of SWNT nucleation on Fe catalysts (Ohta et al., 2009).

a

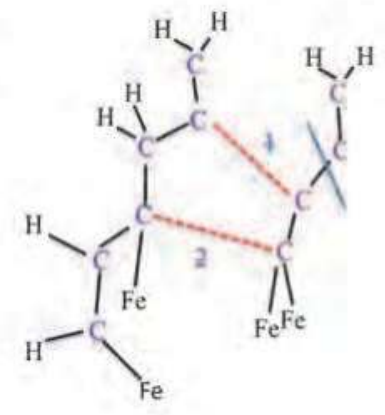

b

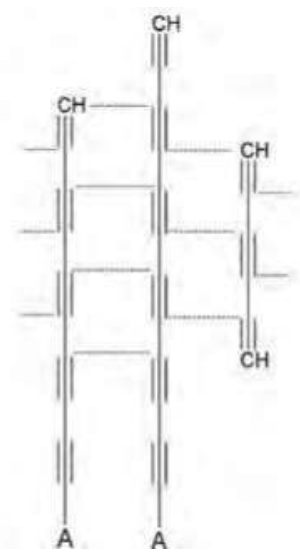

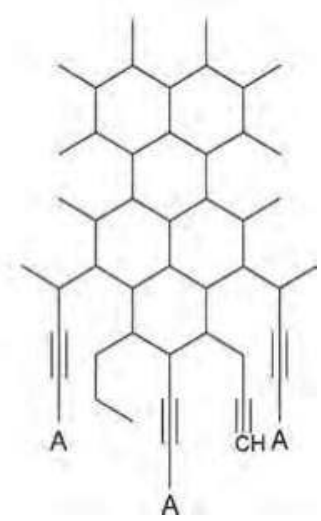

Fig. 2. Oligomerisation, or cross-linking, of extended polyyne chains on the catalyst surface leads to carbon ring formation. a) Explicit example of cross-linking resulting in pentagonal ring formation observed in QM/MD simulations. Red lines indicate newly formed C-C bonds, blue lines indicate broken C-C bonds. Number 1or 2 indicates reaction step. b) Crosslinking reaction proposed by Eres (Eres et al., 2009), resulting in the formation of a graphenetype structure. (Reprinted with permission. (C 2009 American Chemical Society)

In the present approach, the hydrogen was simply removed from the gas-phase carbonaceous molecules prior to their interaction with the catalyst nanoparticle. Fig. 4 depicts this adsorption process, and the process of constant temperature annealing that followed. In this case, the MD relaxation of this $\mathrm{Fe}_{38}$-carbon system was continued for 410 ps. Here $\mathrm{C}_{2}$ moieties have been employed, which are considered to be essentially equivalent with the gas-phase acetylene feedstock molecules discussed in \$3.1. However, the absence of hydrogen here is not unrealistic, considering the known products resulting from the vaporisation of graphite/graphene via arc-discharge or laser-ablation processes. In addition, the use of $\mathrm{C}_{2}$ allowed the atomistic mechanism of SWNT nucleation to be probed more easily than before. Fig. 4a shows that this SWNT nucleation process may be partitioned into three distinct stages. To begin with, $\mathrm{C}_{2}$ units adsorbed onto the $\mathrm{Fe}_{38}$ catalyst surface from the gas-phase. The relatively weak $\mathrm{Fe}-\mathrm{C}$ interaction energy facilitated the subsequent diffusion of these $C_{2}$ units over the catalyst surface. As a natural consequence of this diffusion, $C_{2}$ units ultimately began to interact with each other, coalescing to form longer polyyne chains (i.e. $\mathrm{Fe}-\mathrm{C}_{n}$-Fe structures) on/over the catalyst surface. This was apparently the rate-limitingstep of the nucleation process, in that it was ca. 100 ps before the second stage of the nucleation mechanism took place (Fig. 4b). This second stage featured the initial ring condensation processes on the catalyst surface, while the subsequent third stage consisted entirely of additional ring condensation events, resulting in the formation of a SWNT capfragment. The exact mechanism of this initial ring condensation process is depicted in Fig. 4c. Fig. $4 \mathrm{~b}$ shows that there was generally a preference for the formation of pentagonal rings, as opposed to hexagonal or heptagonal rings, during the initial stages of SWNT nucleation. 
Indeed, the initial ring structure formed was pentagonal. This fact is consistent with knowledge regarding the formation mechanism of fullerenes at high temperatures (Irle et al., 2006). The longevity of these pentagonal rings, however, is attributed to the high positive curvature of the catalyst nanoparticle surface (due to its small diameter) (Fan et al., 2003). In effect, the growing $s p^{2}$-hybridised carbon network attempts to 'mould' itself to its supporting catalyst substrate from its very beginnings.

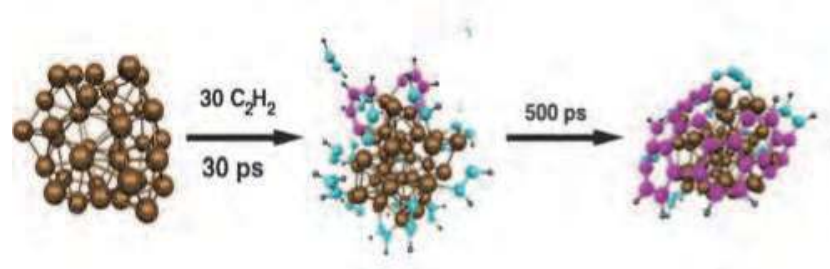

(i)

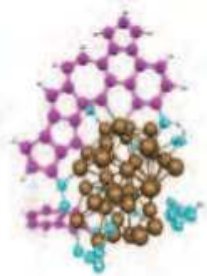

(ii)

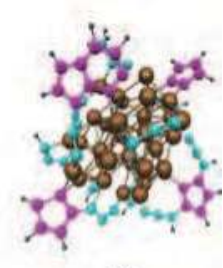

(iii)

Fig. 3. An extended $s p^{2}$-hybridised carbon network is formed on the catalyst surface following polyyne oligomerisation. QM/MD simulations indicate that structures (i), (ii) and (iii), are typically formed. Structure (i) is akin to the 'yarmulke' SWNT cap fragment proposed by Smalley et al. (Dai et al., 1996). Structure (ii) is typically formed in the presence of higher $\mathrm{H}$ concentrations, and points to the possibility that SWNT nucleation may take place in the absence of a SWNT cap fragment. Structure (iii) features a catalyst nanoparticle covered with $s p^{2}$-hybridised carbon 'islands'. Color conventions as in Fig. 1; pink spheres represent $s p^{2}$-hybridised carbon atoms.

The initial pentagonal ring observed in Fig. 4 acted as an anchor, or cornerstone, for all subsequent ring condensation events. This period of ring condensation (stage three of the nucleation process) consisted of a periodic process (Fig. $4 \mathrm{~d}$ ) in which adjacent polyyne chains interacted on the catalyst surface due to their diffusion, thus extending the $s p^{2}-$ hybridised carbon structure. This process is best illustrated by the initial ring condensation event, in which two adjacent polyyne chains coalesced, resulting in a 'Y-junction'. This initial $s p^{2}$-hybridised carbon atom was, in essence, the nucleus of the final SWNT itself, since all subsequent ring condensation was based around it. The first pentagonal ring formed following the sinusoidal-type diffusion of the two arms of the Y-junction. With respect to the original $s p^{2}$-hybridised carbon atom, the most energetically favorable interaction corresponded to the interaction between the second carbon atoms of each arm. This therefore explains the observed preference for pentagonal ring formation observed during the initial stages of SWNT nucleation. It is noted, however, that this interaction was only the most favorable due to the approximate $120^{\circ}$ bond angle provided by the single $s p^{2}-$ hybridised carbon atom. In subsequent ring condensation events, this single carbon atom was often replaced with a C-C moiety, thus this bond angle was modified (or removed entirely). The most energetically favorable interaction therefore corresponded to that between carbon atoms at varying positions along the arms of the Y-junction structures.

As will be discussed in $\S 4$, extended polyyne chains play a dominant role not only in SWNT nucleation, but also in the subsequent 'continued' growth of SWNT structures. In this sense, SWNT growth is therefore very similar to the self-assembly of fullerenes (Irle et al., 2006). This leads to the conclusion that such polyyne chains are essential for both the conception and the extension of any $s p^{2}$-hybridised carbon network. Of course, the crucial difference 
between SWNT and fullerene nucleation here is the presence of the catalyst nanoparticle. In particular, it is noted here that the QM/MD simulation presented in Fig. 4 once again verifies the original proposal of Smalley et al., i.e. that one of the fundamental roles of the catalyst nanoparticle here is preventing the closure of the growing $s p^{2}$-hybridised structure (Thess et al., 1996).

a

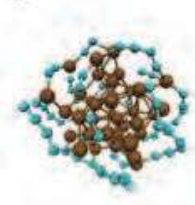

0 ps

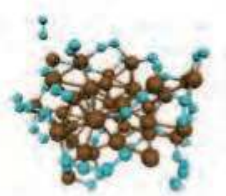

100 ps

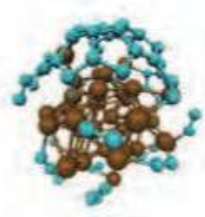

200 ps

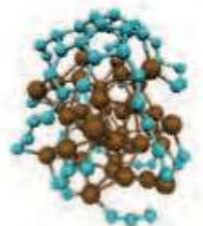

300 ps

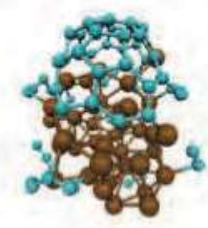

410 ps

b
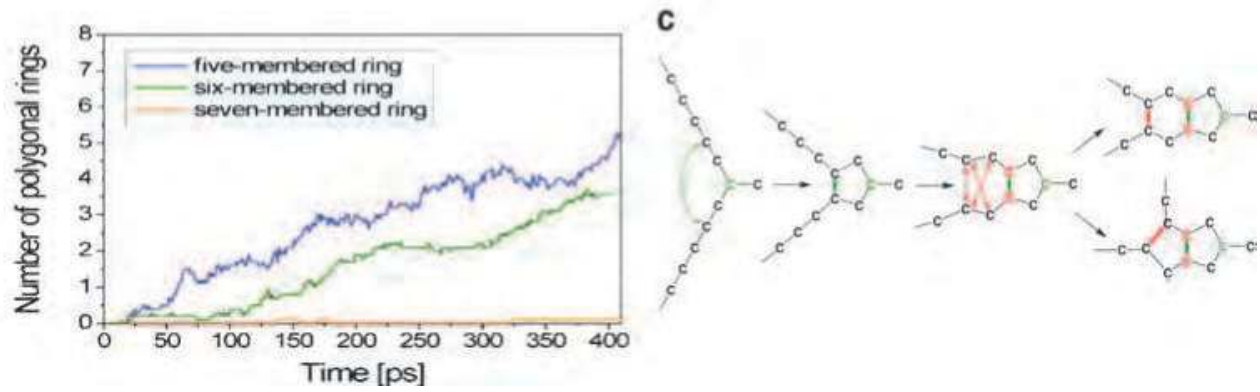

Fig. 4. SWNT nucleation occurs via three distinct stages, according to QM/MD simulations. a) QM/MD relaxation of a $30 \mathrm{C}_{2}-\mathrm{Fe}_{38}$ model system at $1500 \mathrm{~K}$ yields a distinct SWNT cap fragment after 410 ps. Color conventions as in Fig. 1. b) SWNT nucleation is driven by successive ring condensation events on the catalyst surface. The preferential formation of pentagonal rings in this structure is attributed to the curvature of the catalyst surface, and the diffusion dynamics of extended polyyne chains. c) The SWNT nucleus. A single $s p^{2}-$ hybridised carbon atom acts as the cornerstone of all subsequent ring formation events in the nascent SWNT structure. (Adapted from (Ohta et al., 2009). Reprinted with permission. (C) 2009 American Chemical Society)

\subsection{SWNT nucleation from amorphous Fe \& Ni carbide precursors}

According to the VLS mechanism, CNT nucleation and growth are preceded by a gaseous carbon/catalyst phase which co-condense forming a catalyst-carbide nanoparticle. Images of transition metal carbide nanoparticles have been obtained using transmission electron microscopy on several occasions (Yoshida et al., 2008; Yoshida et al., 2009) (see Fig. 5). Yet, to date there is no experimental evidence indicating that this carbide phase necessarily precedes the nucleation and growth of SWNTs. Indeed, QM/MD simulations discussed in \$3.1-3.2 indicate that, for nanoparticle catalysts of $c a .1 \mathrm{~nm}$, a carbide phase is not formed at $1500 \mathrm{~K}$. The thermodynamic stability of bulk transition metal carbide nanoparticles have also been drawn into question from a number of independent approaches. Assumedly then, SWNT nucleation may occur in the absence of a carbide phase. Such disparities between theoretical 
and experimental assertions give reason for further study of the role of the carbide phase with respect to SWNT nucleation and growth.

QM/MD simulations of SWNT nucleation from amorphous $\mathrm{Fe}$ - and Ni-carbide nanoparticles at $1400 \mathrm{~K}$ are depicted in Fig. 6. It is evident from this figure that, upon MD relaxation at constant temperature, the amorphous carbide phase almost immediately decomposes (within ca 5 - 10 ps), yielding segregated Fe/Ni-carbon systems. This phenomenon is known to take place regardless of temperature, or the carbon concentration in the amorphous carbide phase (Page et al., 2010d). Fig. 6 also indicates that the SWNT nucleation mechanism in this case (from a Ni-carbide) is the same as that presented in \$3.13.2. For example, the almost immediate precipitation of carbon from the nanoparticle bulk to the surface leads to the formation of extended polyyne chains over the nanoparticle surface. The oligomerisation/cross-linking etc. of these chains then leads to the formation of primarily pentagonal and hexagonal rings (Fig. 6a) as the SWNT cap fragment is formed. The initial ring structure in all cases here is invariably a pentagonal ring. Thus, the SWNT nucleation mechanism on $\mathrm{Fe} / \mathrm{Ni}$ catalysts is evidently independent of the type of metal catalyst, temperature, and origin/type of the feedstock carbon employed. While the fact that SWNT nucleation originates from a Ni-carbide structure is not unexpected (since it has been predicted in several prior REBO-based MD investigations (Shibuta \& Maruyama, 2002; 2003)), what is remarkable is the invariance of the SWNT nucleation mechanism with respect to these pertinent experimental factors.

Despite this invariance, differences in the ultimate product of this nucleation process are evident with respect to both the catalyst type and the simulation temperature. The kinetics of SWNT nucleation was also affected by the type of catalyst employed - explicitly, SWNT nucleation from the decomposition of Ni-carbide proceeded more quickly, compared to that from Fe-carbide (see Fig. 6 and Fig. 7). It was observed that at higher temperatures (2000 K, as opposed to 800 or $1400 \mathrm{~K}$ ), the populations of pentagonal and hexagonal rings in the SWNT cap fragment were approximately equal. On the other hand, at lower temperatures a distinct preference towards pentagonal ring formation existed. These differing ring populations were ascribed to the effect of temperature on the SWNT nucleation dynamics. At higher temperatures, the growing polyyne chains on the catalyst surface are more thermally excited, and thus exhibited larger amplitude vibrational motion. Considering the pentagonal ring mechanism given in Fig. 4c, this increased motion makes the formation of a $\mathrm{C}-\mathrm{C}$ bond between tertiary carbon atoms (with respect to the $s p^{2}$-hybridised 'cornerstone' carbon atom) more likely. Hence, hexagonal ring formation is more probable in this case. Perhaps the most important difference observed between the kinetics of SWNT nucleation from $\mathrm{Fe}$ - and Ni-carbide nanoparticles, however, pertains to the relative rates of SWNT nucleation. It was recently established that SWNT nucleation is significantly more labile on Ni catalysts, compared to Fe catalysts. This observation may be directly attributed to the relative strengths of the catalyst-carbon interactions. This point will be a recurring theme throughout the present work, as it dominates many aspects of both SWNT nucleation and growth. For example, the catalyst-carbon interaction strengths, calculated using SCC-DFTB, are 1.78 and $1.06 \mathrm{eV}$ for $\mathrm{Fe}-\mathrm{C}$ and $\mathrm{Ni}-\mathrm{C}$, respectively. For comparison, the $\mathrm{C}-\mathrm{C}$ interaction strength is $9.14 \mathrm{eV}$. Therefore, $\mathrm{Fe}-\mathrm{C}$ bond formation is more favorable than $\mathrm{Ni}-\mathrm{C}$ bond formation, in a thermodynamic sense. Consequently, C-C bond formation during the decomposition of Fe-carbide nanoparticles is impeded, which in turn impedes the nucleation of the $s p^{2}$-hybridised carbon network. This argument also explains other phenomena related to 
SWNT nucleation, such as the lifetimes of the bulk and subsurface carbide intermediate species (Page et al., 2010d) (see Fig. 7). In particular, the average time required for

a

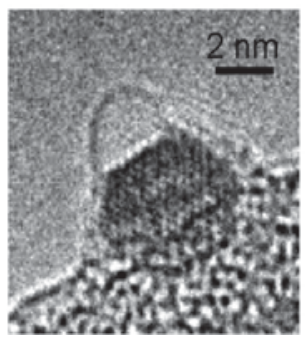

b

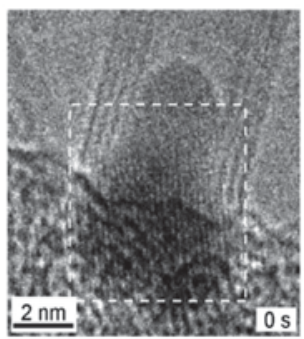

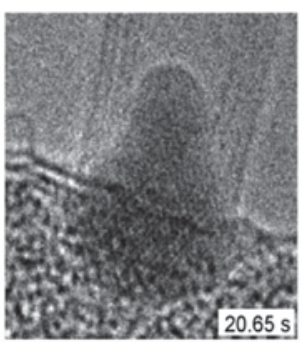

Fig. 5. TEM images of intermediate structures observed during SWNT growth experiments. a) A SWNT cap-fragment bound to a crystalline $\mathrm{Fe}_{3} \mathrm{C}$ nanoparticle with an approximate diameter of $2 \mathrm{~nm}$. (Adapted from (Yoshida et al., 2008). Reprinted with permission. (C) 2008 American Chemical Society) b) A MWNT growing from a $(\mathrm{Fe}, \mathrm{Mo}){ }_{23} \mathrm{C}_{6}$ nanoparticle catalyst. In this case the nanoparticle diameter is ca. $6 \mathrm{~nm}$. (Adapted from (Yoshida et al., 2009). Reprinted with permission. (C) 2009 American Chemical Society)

a

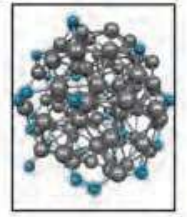

0 ps

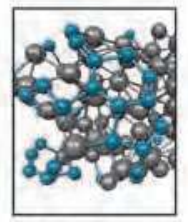

$121.04 \mathrm{ps}$

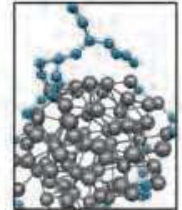

$96.08 \mathrm{ps}$

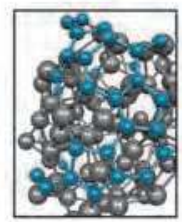

$139.08 \mathrm{ps}$

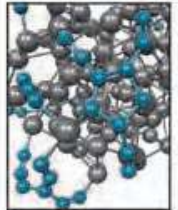

$116.84 \mathrm{ps}$

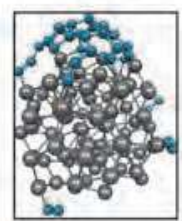

$300.00 \mathrm{ps}$ b

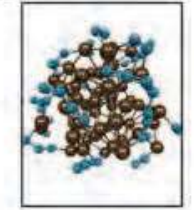

240.00 ps

C

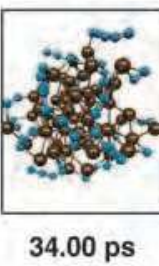

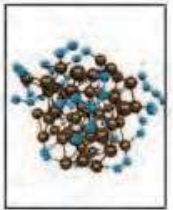

$246.84 \mathrm{ps}$

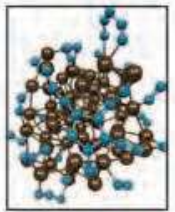

$34.20 \mathrm{ps}$

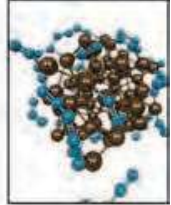

$252.36 \mathrm{ps}$

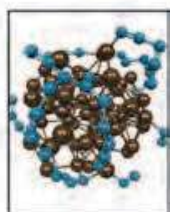

$43.28 \mathrm{ps}$

Fig. 6. The kinetics of SWNT nucleation from Ni-carbide are enhanced relative to those from Fe-carbide. Nevertheless, the mechanism of SWNT nucleation is identical in both cases. a) The evolution of a computed SWNT nucleation trajectory from a $\mathrm{Ni}_{77} \mathrm{C}_{39}$ carbide nanoparticle at $1400 \mathrm{~K}$. The SWNT cap fragment is formed within 300 ps following repeated ring condensation events. b), c) The evolution of two $\mathrm{Fe}_{58} \mathrm{C}_{58}$ carbide nanoparticles at $1400 \mathrm{~K}$. The stronger Fe-C interaction impedes the formation of $\mathrm{C}-\mathrm{C}$ bond, and therefore impedes the SWNT nucleation process itself. Both trajectories show examples of the formation, and subsequent destruction, of pentagonal carbon rings. Color conventions as in Fig. 1; grey spheres represent Ni atoms. (Adapted from (Page et al., 2010d). Reprinted with permission. (C) 2010 American Chemical Society) 
precipitation of all carbon from the nanoparticle bulk to the nanoparticle surface/subsurface in the case of Fe-carbide always exceeds that for Ni-carbide, except at low temperatures. At $800 \mathrm{~K}$, the reverse is the case, since the Ni-carbide nanoparticle exists in the solid phase, while the Fe-carbide nanoparticle is liquid. These QM/MD findings therefore support recent claims that a subsurface carbide structure (in which a high density of carbon exists at, or just below, the nanoparticle surface) precedes SWNT nucleation and growth (Amara et al., 2006; 2008b; a; Harutyunyan et al., 2008; Amara et al., 2009).
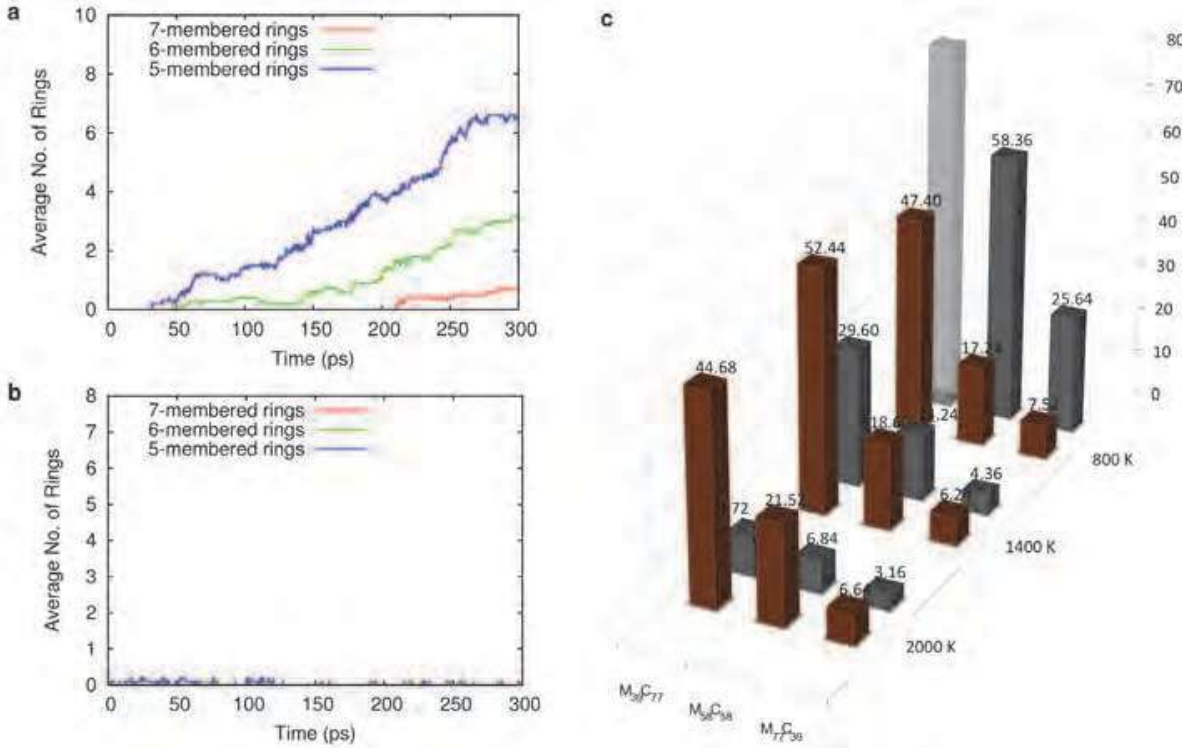

Fig. 7. The kinetics of SWNT nucleation from Ni-carbide are enhanced relative to those from Fe-carbide. Average polygonal carbon rings formed from a) $\mathrm{Ni}_{58} \mathrm{C}_{58}$ and b) $\mathrm{Fe}_{58} \mathrm{C}_{58}$ at $1400 \mathrm{~K}$. c) Average carbon precipitation times (in ps) for Fe- and Ni-carbide nanoparticles between 800 and $2000 \mathrm{~K}$. Fe-carbide carbon remains within the nanoparticle bulk for a longer time period, compared to Ni-carbide carbon, due to the stronger Fe-C interaction. At $800 \mathrm{~K}$ the trend is reversed since $\mathrm{Ni}$-carbide exists in the solid phase. All data averaged over 10 trajectories. Brown and grey columns refer to Fe- and Ni-carbide data, respectively. Transparent columns indicate precipitation times greater than 300 ps. (Adapted from (Page et al., 2010d). Reprinted with permission. (C) 2010 American Chemical Society)

\subsection{A new breed of catalysts: SWNT nucleation on $\mathrm{SiO}_{2}, \mathrm{SiC}$ and $\mathrm{Si}$}

The mechanism of SWNT nucleation on traditional, transition metal catalysts such as $\mathrm{Fe}, \mathrm{Ni}$ and Co has now been the subject of both experimental and theoretical scrutiny for approximately a decade. Since 2009, however, a number of experimental reports (Takagi et al., 2007; Liu et al., 2008; Bachmatiuk et al., 2009; Homma et al., 2009; Huang et al., 2009; Liu et al., 2009a; Liu et al., 2009b; Liu et al., 2010a; Liu et al., 2010b) have established non-traditional nanomaterials to be catalytically active in the context of SWNT nucleation and growth from methane and ethanol CVD. Si-based materials, and in particular $\mathrm{SiO}_{2}$, have been remarkably 
successful in this respect. Yet it has only been since 2011 that the atomistic mechanisms of $\mathrm{SiO}_{2-}, \mathrm{SiC}$ - and Si-catalysed SWNT nucleation have been established. These QM/MD investigations will be the focus of this section.

QM/MD simulations of methane CVD on $\mathrm{SiO}_{2}$ nanoparticles at $1200 \mathrm{~K}$ (Page et al., 2011b) is outlined in Fig. 8. Due to the inherently low catalytic activity of $\mathrm{SiO}_{2}$ itself, $\mathrm{CH}_{x}$ radicals $(x=$ $0-3$ and is chosen randomly) were supplied to the $\mathrm{SiO}_{2}$ instead of $\mathrm{CH}_{4}$. This approach was motivated by the prior conclusion that $\mathrm{CH}_{4}$ decomposes pyrolitically prior to adsorption on the $\mathrm{SiO}_{2}$ surface (Liu et al., 2009b). In contrast to CVD using traditional transition-metal catalysts, a complex chemical process was observed on $\mathrm{SiO}_{2}$. Most notably, $\mathrm{CO}$ was produced as the primary chemical product via the carbothermal reduction of the $\mathrm{SiO}_{2}$ nanoparticle, a fact that is consistent with recent experimental observations (Bachmatiuk et al., 2009). The production of each $\mathrm{CO}$ molecule first required hydrogen-abstraction from neighboring $\mathrm{C}, \mathrm{Si}$ or $\mathrm{O}$ atoms. Ultimately, the insertion of carbon into/removal of oxygen from the $\mathrm{SiO}_{2}$ nanoparticle resulted in the local formation of amorphous $\mathrm{SiC}$. However, this carbothermal reduction was limited to the outer regions of the catalyst, with the core of the

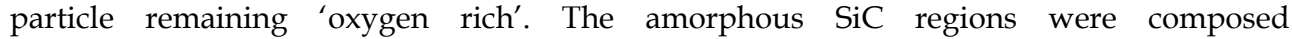
predominantly of extended polyyne chains 'anchored' in place by native Si atoms.

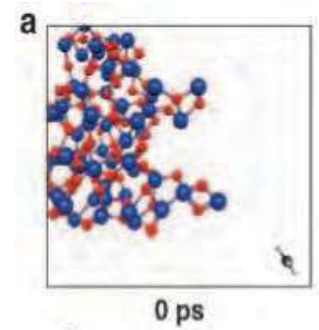

b

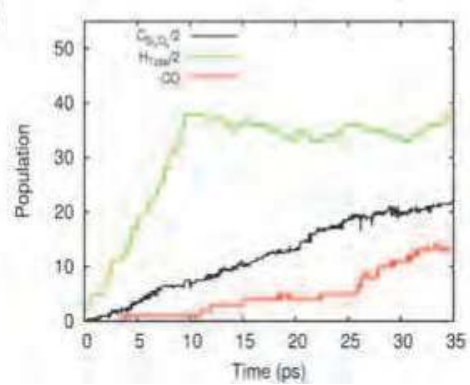

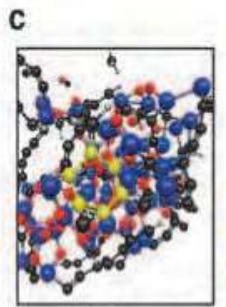

$75.0 \mathrm{ps}$

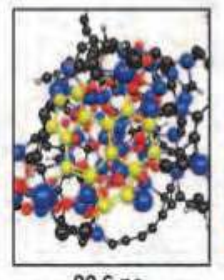

$92.6 \mathrm{ps}$

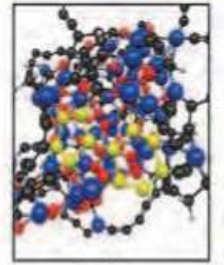

$91.8 \mathrm{ps}$

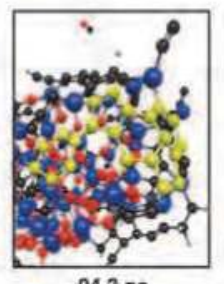

94.3 ps

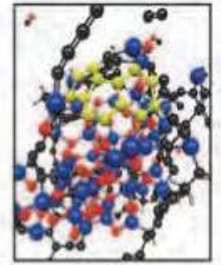

$92.3 \mathrm{ps}$

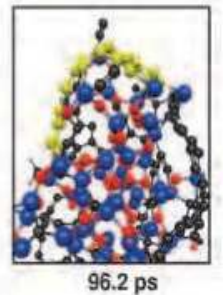

Fig. 8. $\mathrm{CH}_{4} \mathrm{CVD}$ on $\mathrm{SiO}_{2}$ nanoparticles at $1200 \mathrm{~K}$ leads to SWNT nucleation via a VSS mechanism. a) Snapshots at 0 and 35 ps showing the CVD process. b) $\mathrm{CO}$ is the major chemical product of the $\mathrm{CH}_{4} \mathrm{CVD}$ process. The production of $\mathrm{CO}$ first requires the natural removal of $\mathrm{H}$ from the $\mathrm{CO}$ carbon atom. $\mathrm{C}_{\mathrm{SixOy}}$ and $\mathrm{H}_{\mathrm{SixOy}}$ are the concentration of $\mathrm{C}$ and $\mathrm{H}$ on the $\mathrm{SiO}_{2}$ nanoparticle, respectively. c) Evolution of SWNT nucleation on $\mathrm{SiO}_{2}$ nanoparticles. Contrary to nucleation on transition-metal catalysts, nucleation here requires the saturation of the solid-phase catalyst with carbon. Blue, red and black spheres represent $\mathrm{Si}, \mathrm{O}$ and $\mathrm{C}$, respectively. Yellow spheres represent $\mathrm{C}$ atoms involved in SWNT nucleation. (Adapted from (Page et al., 2011b). Reprinted with permission. (C) 2011 American Chemical Society) 
Consequently, these polyyne chains exhibit restricted vibrational and translational mobility, compared to the equivalent precursor structures observed during transition-metal catalysed SWNT nucleation. A more detailed discussion of the thermodynamic reasons underpinning these phenomena is given below. At high concentrations of surface polyyne chains SWNT nucleation was observed. This observation supports the previous claim by Homma and coworkers that SWNT nucleation on solid, covalent catalysts requires a 'carbon-covered' catalyst nanoparticle in order for nucleation to take place (Homma et al., 2009). The pentagonal-ring-first mechanism, established by QM/MD simulations and discussed in \$3.1-3.3, therefore played no role in the current context. Similarly, the liquid carbide phase that is central to the VLS mechanism of SWNT, discussed in §3.3, is absent in the case of $\mathrm{SiO}_{2}$-catalysed SWNT nucleation. This conclusion followed an analysis of the instantaneous Lindemann index (Lindemann, 1910) of the $\mathrm{SiO}_{2}$ nanoparticle during the CVD process. At all times, the Lindemann index revealed that the $\mathrm{SiO}_{2}$ nanoparticle existed as a solid phase structure. Moreover, QM/MD relaxation of this nanoparticle at elevated temperatures (up to $3000 \mathrm{~K}$ ) indicated that nanoparticle $\mathrm{SiO}_{2}$ decomposes from the solid phase at sufficiently high temperatures (Page et al., 2011a). This sublimative phenomenon here rules out the VLS mechanism as an explanation of $\mathrm{SiO}_{2}$-catalysed SWNT nucleation and growth entirely. Instead, QM/MD simulations point to a vapor-solid-solid (VSS) mechanism explaining SWNT nucleation and growth in this case. The mechanisms of SWNT nucleation and growth on traditional and non-traditional catalysts are therefore of fundamentally different natures. Subsequent experimental results (Liu et al., 2011) have since corroborated this proposed VSS mechanism.

The observation that the catalytically relevant region of the $\mathrm{SiO}_{2}$ nanoparticle is effectively devoid of oxygen motivated the subsequent QM/MD investigation of SWNT nucleation on pure Si nanoparticles. To this end, a $\mathrm{Si}_{58}$ nanoparticle of approximate dimension $0.9 \times 0.9 \times$ $0.9 \mathrm{~nm}^{3}$ was employed as a CVD catalyst at 1200 and $1800 \mathrm{~K}$. Gas-phase $\mathrm{C}_{2}$ moieties were adsorbed on the surface of this catalyst nanoparticle in the manner described in \$3.2. Two different concentrations of carbon, viz. 30 and 100, were employed here, following the observation made regarding the dependence of SWNT nucleation on surface carbon concentration using $\mathrm{SiO}_{2}$ catalyst nanoparticles. The structures of these $\mathrm{Si}_{58} \mathrm{C}_{60}$ and $\mathrm{Si}_{58} \mathrm{C}_{200}$ model systems, following 100 and 45 ps, are given in Fig. 9. Upon adsorption on the $\mathrm{Si}_{58}$ surface, these $\mathrm{C}_{2}$ moieties generally coalesced, forming extended polyyne chains, in an identical fashion to nucleation on $\mathrm{Fe}, \mathrm{Ni}$ and $\mathrm{SiO}_{2}$ catalysts. However, the mobility of these polyyne chains in the case of $\mathrm{Si}_{58}$ was notably restricted, as was observed in the case of $\mathrm{SiO}_{2}$. This was also the case at a higher annealing temperature of $1800 \mathrm{~K}$, leading to the conclusion that the effect of temperature (at least below $2000 \mathrm{~K}$ ) on this SWNT nucleation process was effectively negligible. It was noted that this was not the case at even higher temperatures, as will be discussed below in the context of SWNT nucleation from SiC. Once formed, these polyyne chains themselves gradually coalesced on the nanoparticle surface, ultimately forming extended branched carbon networks. While this is consistent with the initial steps in SWNT nucleation discussed in \$3.1-3.3 in an atomistic sense, it is noted that the kinetics of this coalescence on $\mathrm{Si}_{58}$ is significantly slower, compared to traditional, transition metal catalysts. In particular, in the latter case the rate-limiting step of SWNT nucleation may be considered to be the formation of the SWNT 'nucleus' (the initial polygonal carbon ring structure). Following the formation of this structure, the subsequent ring condensation and cap-formation process proceeds relatively quickly. This is not so in the presence of $\mathrm{Si}$ nanoparticle catalysts. Fig. 10a shows that, following the formation of the SWNT nucleus on 
a

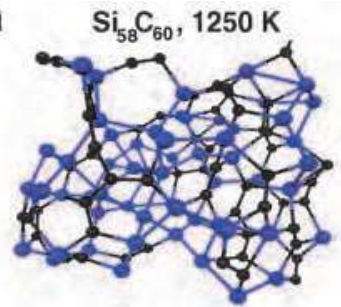

$\mathrm{Si}_{58} \mathrm{C}_{200}, 1250 \mathrm{~K}$

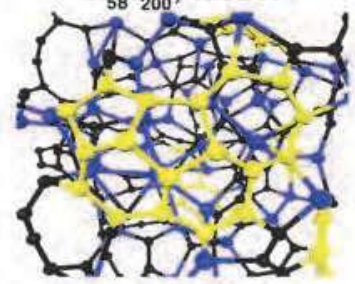

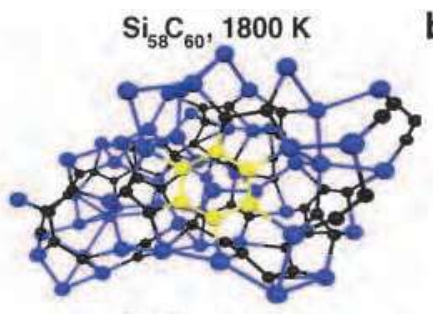

$\mathrm{Si}_{58} \mathrm{C}_{200}, 1800 \mathrm{~K}$

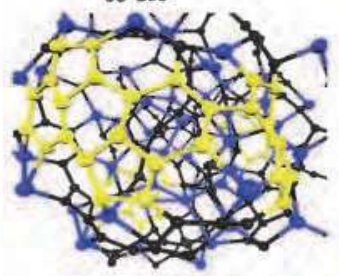

b

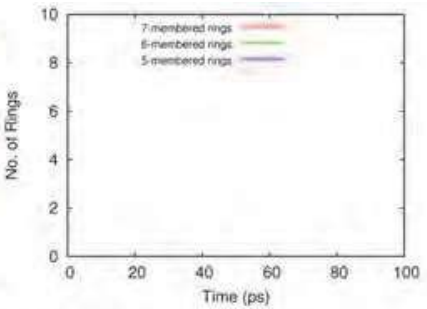

C

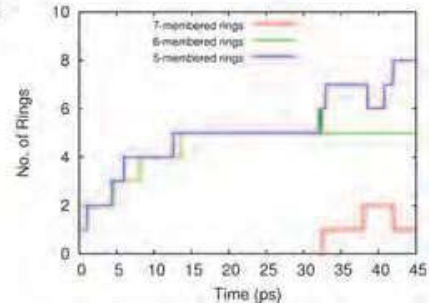

Fig. 9. SWNT nucleation on Si catalyst nanoparticles following the adsorption of gas-phase $\mathrm{C}_{2}$. a) Structures of $\mathrm{Si}_{58} \mathrm{C}_{60}$ and $\mathrm{Si}_{58} \mathrm{C}_{200}$ model complexes at 1200 and $1800 \mathrm{~K}$. Color conventions as in Fig. 8. b) Polygonal ring populations observed using low [C] conditions (i.e. a $\mathrm{Si}_{58} \mathrm{C}_{60}$ model system). c) Polygonal ring populations observed using high [C] conditions (i.e. $\mathrm{Si}_{58} \mathrm{C}_{200}$ model system). It is evident that the initial saturation of the Si catalyst surface with carbon is necessary in order for SWNT nucleation to proceed.

$\mathrm{Si}_{58}$, the subsequent extension of the $s p^{2}$-hybridised carbon network proceeded at a significantly slower rate. Fig. 9a also illustrates the effect of surface carbon concentration on SWNT nucleation. For example, the formation of polygonal carbon rings in the $\mathrm{Si}_{58} \mathrm{C}_{60}$ complex (following the adsorption of $30 \mathrm{C}_{2}$ species) is limited to a single hexagonal ring structure after 100 ps. Conversely, an extended network of carbon ring structures was formed in the $\mathrm{Si}_{58} \mathrm{C}_{200}$ model complex after only $50 \mathrm{ps}$. Thus, as was the case regarding $\mathrm{SiO}_{2}$

a

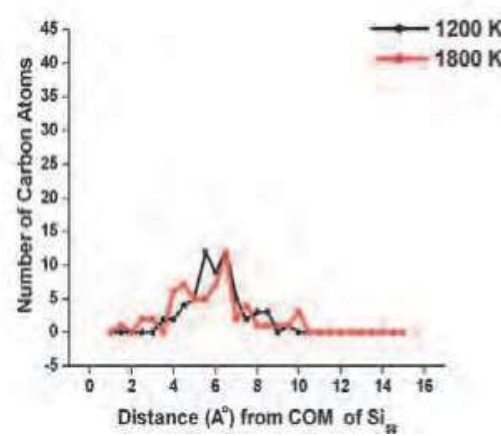

b

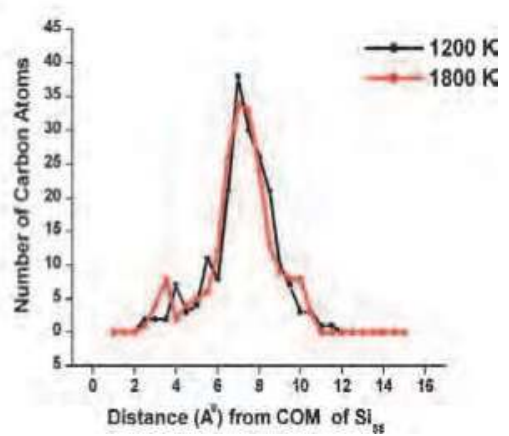

Fig. 10. Radial distributions of carbon in a) $\mathrm{Si}_{58} \mathrm{C}_{60}$ and b) $\mathrm{Si}_{58} \mathrm{C}_{200}$ model complexes at 1200 and $1800 \mathrm{~K}$. The inability of carbon to freely diffuse through the bulk region of the $\mathrm{Si}$ nanoparticle is evident. Consequently, the majority of the carbon in both cases resides on the nanoparticle surface, the latter of which is solid. SWNT nucleation cannot therefore proceed via a VLS mechanism. 
catalyst nanoparticles, it is evident that the saturation of the Si nanoparticle surface with carbon is also a prerequisite for SWNT nucleation in this case. In this sense then, SWNT nucleation on $\mathrm{SiO}_{2}$ and $\mathrm{Si}_{58}$ seemingly proceeds via an identical route - this point will be discussed at greater length below.

Following the adsorption of $\mathrm{C}_{2}$ onto the $\mathrm{Si}_{58}$ nanoparticle surface, the resultant surface structure resembled an amorphous $\mathrm{SiC}$ phase, while the core of the Si nanoparticle remained pristine. This is evident from Fig. 10a, which shows the radial distribution of carbon within the Si nanoparticle as SWNT nucleation proceeds. This figure also shows that, at higher temperature, the penetration of the Si nanoparticle by adsorbed carbon atoms becomes more probable, and is independent of the surface carbon concentration. Yet, the free diffusion of carbon through the nanoparticle bulk and surface in this case is restricted below $2000 \mathrm{~K}$. The latter observation may be explained with recourse to an analysis of the nanoparticle phase during SWNT nucleation. This is conveniently done in the realm of QM/MD simulations via the Lindemann index (Lindemann, 1910), $\delta$,

$$
\delta=\frac{1}{N} \sum_{i} \delta_{i}
$$

where,

$$
\delta_{i}=\frac{1}{N-1} \sum_{j \neq i} \frac{\sqrt{\left\langle r_{i j}^{2}\right\rangle_{T}-\left\langle r_{i j}\right\rangle_{T}^{2}}}{\left\langle r_{i j}\right\rangle_{T}}
$$

Here, $N$ is the number of atoms in the relevant system, $r_{i j}$ is the instantaneous distance between atoms $i$ and $j$, and the brackets denote thermal averaging over a finite interval of time at temperature $T$. It is noted here that $\delta$ describes all atoms in the system, and is thus generally referred to as the 'global' Lindemann index. On the other hand, $\delta_{i}$ pertains only to the motion of atom $i$, and is therefore referred to as the 'atomic' Lindemann index. In the current discussion, we will make reference to both $\delta$ and $\delta_{i}$. The Lindemann index has been used with particular success in the investigation of transition and main group metal species (both bulk and nanoparticle structures) (Ding et al., 2006b; Puri \& Yang, 2007; Neyts \& Bogaerts, 2009; Wen et al., 2009). From these investigations, the efficacy of the Lindemann index in the prediction of nanoparticle melting points has been established. For example, it is now generally accepted that the 'threshold' $\delta$ value, which signifies the transition between the solid and liquid phases is between 0.10 - 0.15 (Ding et al., 2006b; Puri \& Yang, 2007; Neyts \& Bogaerts, 2009; Wen et al., 2009). Thus, any system exhibiting a $\delta$ below this threshold value may be considered to be solid, whereas those with $\delta$ above this threshold value are considered to be liquid. In the case of the pristine $\mathrm{Si}_{58}$ catalyst nanoparticle, $\delta$ at 1200 and $1800 \mathrm{~K}$ were 0.298 and 0.372 , respectively. However, upon the adsorption of $C_{2}$ on the $\mathrm{Si}_{58}$ surface, a dramatic decrease in this Lindemann index was observed. At low carbon concentrations (i.e. the $\mathrm{Si}_{58} \mathrm{C}_{60}$ model complex), these same $\delta$ values were 0.093 and 0.231 , while at high concentrations (i.e. the $\mathrm{Si}_{58} \mathrm{C}_{200}$ model complex), they were 0.049 and 0.088 , respectively. This decrease indicates that the phase of the catalyst nanoparticle here changes from a liquid (when pristine) to solid (when carbon-doped). This therefore makes SWNT 
nucleation via the VLS mechanism impossible. Considering this impasse, and the atomistic similarity between SWNT nucleation from $\mathrm{Si}$ and $\mathrm{SiO}_{2}$ nanoparticles, it is apparent that both proceed via the VSS mechanism, as opposed to the VLS mechanism.

The production of an amorphous $\mathrm{SiC}$ nanoparticle following the adsorption of $\mathrm{C}_{2}$ on $\mathrm{Si}$ nanoparticles warranted the further investigation of the possibility of SWNT nucleation from $\mathrm{SiC}$ itself. Moreover, following the conclusion discussed above, viz. that SWNT nucleation on both $\mathrm{SiO}_{2}$ and $\mathrm{Si}$ occur via identical pathways, it is reasonable to anticipate that the same applies in the context of $\mathrm{SiC}$. To this end, we have investigated nucleation of SWNT cap fragments as a result of the constant temperature thermal annealing of SiC nanoparticles alone. In this case, a model $\mathrm{Si}_{96} \mathrm{C}_{96}$ nanoparticle was annealed between 1000 and $3000 \mathrm{~K}$. An example of SWNT nucleation observed at $2500 \mathrm{~K}$ is illustrated in Fig. 11. It is noted that this temperature is approximately that employed in relevant experiments which demonstrate SWNT growth following the decomposition of SiC crystals (Kusunoki et al., 1997). These QM/MD simulations indicated that SWNT nucleation in this case followed the degradation of the $\mathrm{SiC}$ crystalline structure. Indeed, upon annealing even at temperatures as low as $1000 \mathrm{~K}$ a well-defined crystallinity was not evident in the model SiC nanoparticle even after a relatively short simulation time (i.e. 10-20 ps). The result of this degradation was the disruption of C-Si bonds, in favor of C-C bonds, which exhibited free diffusion through/over the $\mathrm{SiC}$ nanoparticle. This diffusion immediately lead to the elongation/oligomerisation of these polyyne chains with high frequency. However, the frequency of these interactions was concomitantly slower at lower temperatures, such as $1000 \mathrm{~K}$. As is evident from Fig. 11a,b, polygonal ring formation followed the initial period in which the oligomerisation of polyyne chains took place. In this case, the initial polygonal ring formation was the result of the diffusion and subsequent interaction of neighboring $\mathrm{C}_{3}$ and $\mathrm{C}_{2}$ species. Fig. $11 \mathrm{~b}$ shows that subsequent ring condensation then proceeded reasonably rapidly, with a definite cap structure being formed within ca. 200 ps. However, following the formation of this cap structure, the population of polygonal rings here then decreased - such a phenomenon has not been observed in the case of traditional, transition metal catalyst nanoparticles. In a kinetic sense, therefore, SWNT nucleation resulting from thermal degradation of $\mathrm{SiC}$ is anticipated to be less favorable, compared to other traditional catalysts. SWNT nucleation, at the atomic level, is essentially no more than the continual formation of C-C bonds. The origin for these inhibited SWNT nucleation kinetics can therefore be found in thermodynamics, which, at high temperatures, dominate SWNT nucleation. In this sense then, SWNT nucleation is in effect a 'thermodynamic sink'. From §3.3, it is evident that thermal annealing of amorphous Fe- and Ni-carbide nanoparticles yielded well-defined SWNT cap structures, similar to those observed here. However, SWNT nucleation from $\mathrm{Fe}$ - and $\mathrm{Ni}$-carbide nanoparticles also resulted in cap structures exceeding the size of those observed using $\mathrm{SiC}$, both on shorter timescales (generally within $\mathrm{ca} .100 \mathrm{ps}$ ) and at lower temperatures (below $2000 \mathrm{~K}$ ). The strengths of the $\mathrm{Fe}-\mathrm{C}$, Ni-C and Si-C interactions are 1.78, 1.06 and $6.29 \mathrm{eV} /$ atom, respectively, at the SCC-DFTB level of theory (Page et al., 2010d). Recall that the strength of the C-C interaction, using SCC-DFTB, is 9.14 $\mathrm{eV} /$ atom. The weaker interaction of the $\mathrm{Fe} / \mathrm{Ni}$ catalyst with carbon therefore correlates directly with an increased rate of SWNT nucleation. Once a C-C bond forms in the latter case, it is rarely broken due to its greater thermodynamic stability (even if it is not the most energetically stable ring structure). On the other hand, the Si-C and C-C interactions are, thermodynamically, comparable to each other. Consequently, C-C bonds are more frequently broken during nucleation on $\mathrm{SiC}$ nanoparticles. 
a

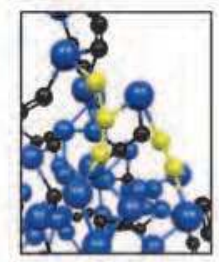

46.02 ps

$\mathrm{C}_{3}-\mathrm{Si}_{-} \mathrm{C}_{4}$

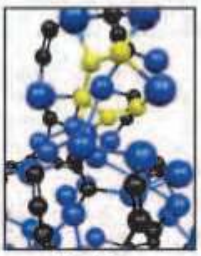

46.52 ps

$\mathrm{C}_{5} \mathrm{Si}$ ring formed. New $C_{4}$ formed.

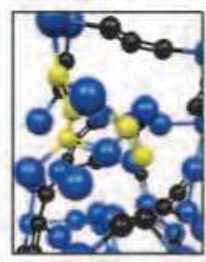

46.52 ps

$\mathrm{C}_{3}-\mathrm{Si}_{-} \mathrm{C}_{2}$ formed. $C_{4}$ unit broken.

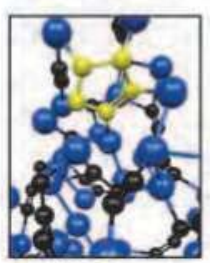

$46.94 \mathrm{ps}$

$C_{5}$ ring formed.

Si atom displaced. b

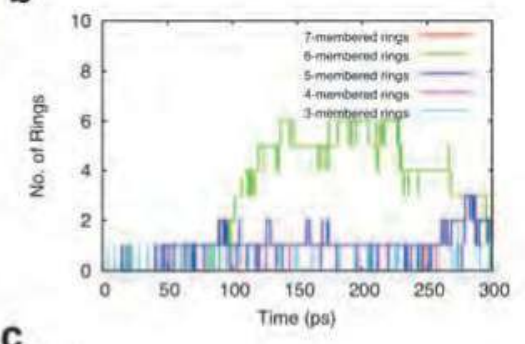

C

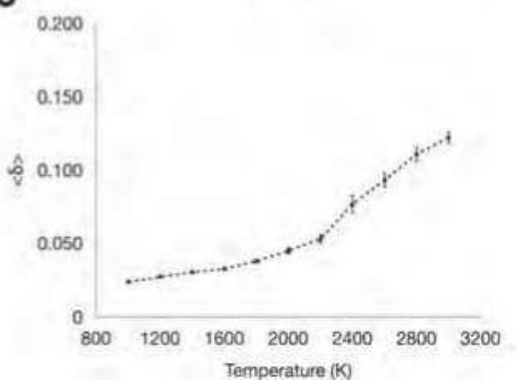

Fig. 11. Thermal annealing at constant temperature $(2500 \mathrm{~K})$ leads to the structural deformation of $\mathrm{SiC}$ nanoparticles, ultimately producing SWNT nucleation. a) The first polygonal ring formation event due to the free diffusion of $\mathrm{C}_{n}$ units within the $\mathrm{SiC}$ nanoparticle. Color conventions as in Fig. 8. b) Polygonal ring formation formed from the structural decomposition of the SiC nanoparticle at $2500 \mathrm{~K}$. c) Time-averaged $\delta$ values of the $\mathrm{SiC}$ nanoparticle between $1000-3000 \mathrm{~K}$, computed over an interval of $50 \mathrm{ps}$. SWNT nucleation below $2600 \mathrm{~K}$ evidently occurs while the $\mathrm{SiC}$ nanoparticle is in the solid phase. Thus, SWNT nucleation can be explained with recourse to a VSS mechanism.

The dependence of $\langle\delta>$ on simulation temperature for the SiC nanoparticle are depicted in Fig. 11c. From this figure it is evident that the $\mathrm{SiC}$ nanoparticle existed in the solid state below $2600 \mathrm{~K}$. However, Fig. 11c suggests that there was undoubtedly some liquid-like character in the $\mathrm{SiC}$ nanoparticle at temperatures above $2600 \mathrm{~K}$. In particular, $\left\langle\delta_{i}\right\rangle$ values (not shown) indicate that, between 1000 and $3000 \mathrm{~K}$, the $\mathrm{SiC}$ nanoparticle exhibited three distinct behaviors depending on the temperature. Firstly, at lower temperatures $(<1400 \mathrm{~K})$ the $\mathrm{SiC}$ nanoparticle were unquestionably solid. At intermediate temperatures (between $1400-2600 \mathrm{~K})$ a gradual increase in $\left\langle\delta_{i}\right\rangle$ for atoms residing close, or near to, the nanoparticle surface was evident. Surface premelting therefore became prevalent at these temperatures, ultimately causing $\langle\delta\rangle$ to increase slightly. Such surface premelting has been shown to be a prominent phenomenon in the melting dynamics of transition metal nanoparticle species (Neyts \& Bogaerts, 2009). In this respect therefore, transition metals and $\mathrm{SiC}$ nanoparticles appear to be equivalent. According to established trends regarding transition metal nanoparticle melting, by increasing the temperature further this surface premelting is followed by the complete liquefaction of the nanoparticle. However, rather than undergoing this solid-liquid phase transition, the SiC nanoparticle instead became quasi-solid at temperatures above $2600 \mathrm{~K}$. One probable cause of this unexpected behavior is 
ascribed to the influence of surface chemistry (viz. the formation of C-C bonds, polyyne chains and polygonal carbon rings etc.) on the Lindemann index itself. In extreme cases, the formation of an extended $s p^{2}$-hybridised carbon network on the $\mathrm{SiC}$ nanoparticle surface, in part, solidified the SiC nanoparticle surface, therefore retarding the melting process.

A pronounced similarity is therefore observed regarding the SWNT nucleation mechanisms on $\mathrm{SiO}_{2}, \mathrm{SiC}$ and $\mathrm{Si}$ catalysts. The results discussed here constitute the first evidence of a catalyst independent mechanism with respect to Si-based catalysts. In addition, these results indicate the mechanism of SWNT nucleation on these Si-based catalysts is remarkably different to that established for transition metal catalysts, and centres around a solid phase catalyst nanoparticle. Since the independence of the SWNT nucleation mechanism has been established and accepted in the case of transition metal catalysts, this conclusion is seemingly unremarkable. However, we point out here that with respect to the majority of 'non-traditional' catalysts such as $\mathrm{SiO}_{2}, \mathrm{SiC}, \mathrm{Si}, \mathrm{Al}_{2} \mathrm{O}_{3}, \mathrm{ZrO}_{2}$, and so on, the precise mechanisms of SWNT nucleation remains are in fact unknown at present. Moreover, at first glance there is no reason to suspect that the SWNT nucleation mechanism on such a diverse range of catalyst species should be in any way related, considering their respective physicochemical properties.

\section{QM/MD simulations of SWNT growth}

We now consider the phenomenon of continued SWNT growth. This is generally defined as the extension of the nanotube sidewall (by the addition of newly created polygonal ring structures) parallel to the axis of growth. Note that this process differs from the process of SWNT nucleation, in which the nascent nanotube cap-fragment is formed. This partitioning of what is actually (in reality) a continuous process is somewhat arbitrary. Nonetheless, it has enabled the precise atomistic mechanism of SWNT growth to be identified and studied.

\subsection{SWNT growth on Fe catalysts}

Continued SWNT growth has been modeled using QM/MD simulations on a number of occasions (see (Page et al., 2010c) and references therein). The approach employed in these investigations typically was similar to that described in $\$ 3.2$ (see Fig. 12). Fe-catalyst nanoparticles were thus first annealed at $1500 \mathrm{~K}$, after which 'simulated' gas-phase carbon feedstock (in this case, $\mathrm{C}$ or $\mathrm{C}_{2}$ ) was adsorbed at various rates at the base of the growing SWNT, or onto the nanoparticle surface itself. Two such nanoparticles have been employed, viz. $\mathrm{Fe}_{38}$ and $\mathrm{Fe}_{55}$. In both cases, a model SWNT cap fragment (a $\mathrm{C}_{40}$ cap of $(5,5)$ chirality), or short SWNT segment (depicted in Fig. 12) were employed to approximate a SWNT cap fragment formed in situ (such as that shown in Fig. 4). The effect of the nanoparticle diameter on the mechanism and kinetics of continued SWNT growth has therefore been elucidated. Somewhat unsurprisingly, the increase in nanoparticle diameter from $0.70 \mathrm{~nm}$ $\left(\mathrm{Fe}_{38}\right)$ to $0.94 \mathrm{~nm}\left(\mathrm{Fe}_{55}\right)$ has no effect on the atomistic mechanism of continued SWNT growth. This mechanism is depicted in Fig. 12. From this figure it is evident that, like SWNT nucleation, the continued SWNT growth process was driven by the extension of the $s p^{2}-$ hybridised carbon network. This extension itself was driven by the formation of polygonal carbon rings at the base of the nanotube structure (at the interface between the nanotube and the catalyst nanoparticle), thereby extending the SWNT cap in a unidirectional manner. From Fig. 12 it can be seen that the SWNT growth process took place almost entirely on the 
catalyst surface. Only very rarely did carbon penetrate the catalyst surface and diffuse through the subsurface region. Similarly, carbon was never observed to freely diffuse through the bulk of the catalyst nanoparticle. Unsurprisingly, this behavior was no different from the behavior observed during SWNT nucleation on $\mathrm{Fe}_{38}$, a fact that is attributed to the nanoparticles relatively small diameter, and consequently relatively high surface energy. It is also noted here that $\mathrm{Fe}_{38}$ and $\mathrm{Fe}_{55}$ are both 'magic number' metal clusters, and so exhibit unusual stability compared to other nanoparticles of comparable diameter. The SWNT growth depicted in Fig. 12 is an example growth from a 'floating' catalyst (most similar to that observed during pure VLS processes, such as arc-discharge). However, it is likely that the mechanism of SWNT 'root'/'tip' growth on supported catalyst nanoparticles is similar to that depicted in Fig. 12, since the majority of SWNT growth chemistry is mediated by the nanoparticle surface itself.

a
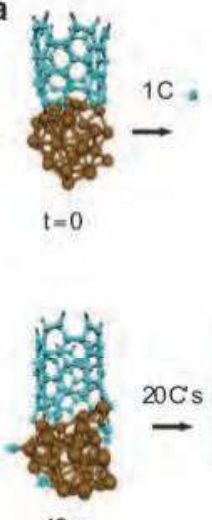

$10 \mathrm{ps}$
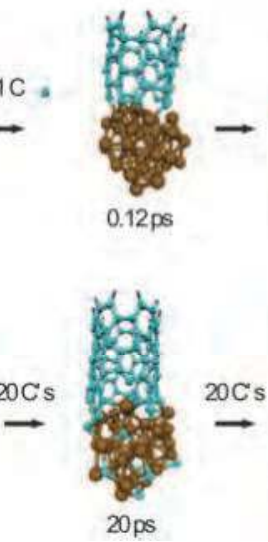

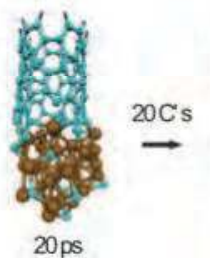

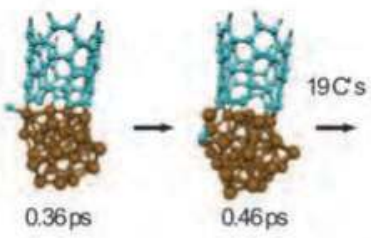

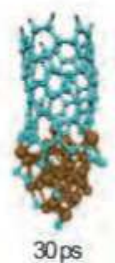

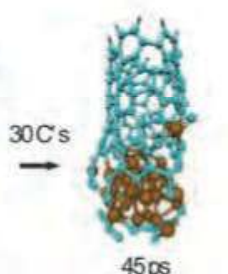

$45 p s$

b

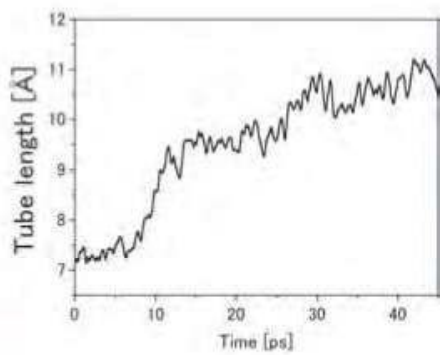

Fig. 12. Continued SWNT growth from a $(5,5)$ SWNT fragment on an $\mathrm{Fe}_{38}$ catalyst nanoparticle at $1500 \mathrm{~K}$. a) The adsorption of gas-phase carbon atoms at a rate of $1 \mathrm{C} / 0.5 \mathrm{ps}$ at the base of the SWNT structure leads to the extension of the $s p^{2}$-hybridised carbon network via the formation of new polygonal rings at the SWNT base. Growth is mediated entirely by the catalyst surface in this case. Color conventions as in Fig. 1. b) The SWNT length as a function of time at $1500 \mathrm{~K}$. Adsorption of gas-phase carbon atoms results in the addition of ca. $4 \AA$ to the base of the SWNT. (Adapted from (Ohta et al., 2008). Reprinted with permission. (C) 2008 American Chemical Society)

While the SWNT growth mechanisms on $\mathrm{Fe}_{38}$ and $\mathrm{Fe}_{55}$ were observed to be the same, this is not so with respect to the kinetics of SWNT growth. QM/MD simulations (Page et al., 2010b) indicate that SWNT growth slows with increasing catalyst nanoparticle diameter - a conclusion that parallels others based on experimental evidence (Huang et al., 2002; Cau et al., 2006; Mora \& Harutyunyan, 2008). This phenomenon is ascribed primarily to the relative surface areas and volumes of the two catalyst nanoparticles. In particular, although the diameter of $\mathrm{Fe}_{55}$ is only slightly larger than that of $\mathrm{Fe}_{38}$, the increases in surface area and volume are more substantial. Thus, the domain over/through which adsorbed $C_{n}$ species may migrate, before being incorporated into the growing SWNT, is concomitantly larger in the case of $\mathrm{Fe}_{55}$. SWNT growth employing the former, smaller catalyst nanoparticle is therefore $c a .19 \%$ faster compared to that on $\mathrm{Fe}_{55}$. It is conceded that both of these growth 
rates exceed those determined experimentally (Puretzky et al., 2002; Futaba et al., 2005; Sharma et al., 2005; Geohegan et al., 2007; Yao et al., 2007; Xiang et al., 2009) by several orders of magnitude. This is a natural consequence of the relatively unnatural carbon adsorption model that has been employed here. Nevertheless, the error thus induced is systematic, and so these relative trends in growth rates remain valid.

\subsection{The importance of interaction energy: Ni versus Fe catalysts}

The fact that different SWNT catalyst materials yield different SWNT growth rates has been established experimentally on numerous occasions (Puretzky et al., 2002; Futaba et al., 2005; Sharma et al., 2005; Geohegan et al., 2007; Yao et al., 2007; Xiang et al., 2009). Nevertheless, no clue was gained as to why this was the case until recently. QM/MD simulations (Page et al., 2010a; Page et al., 2010b) again proved to be of value in this respect, and established the single origin of catalyst-dependent SWNT growth kinetics.

QM/MD simulations of $\mathrm{Ni}_{38}$-catalysed growth from a $\mathrm{C}_{40}$ SWNT cap fragment are summarised in Fig. 13a. Fig. $13 \mathrm{~b}$ shows a comparison of $\mathrm{Fe}_{55}$ - and $\mathrm{Ni}_{55}$-catalysed SWNT growth rates. Once again, in all cases growth was induced by the adsorption of gas-phase carbon atoms at the base of the $\mathrm{C}_{40} \mathrm{SWNT}$ cap structure at a rate of $1 \mathrm{C} / 0.5 \mathrm{ps}$. Comparison of Fig. 12a and 13a shows that the mechanism of SWNT growth, at the atomistic scale, exhibits significant differences. Most notably in this respect is the role of the extended polyyne chains which bridge between the SWNT base and the catalyst surface. In the case of $\mathrm{Fe}_{38}$ (Fig. 12a), these chains generally consisted of 3-4 carbon atoms, and were formed as individual $C / C_{2}$ species diffused across the $\mathrm{Fe}_{38}$ surface towards the SWNT base. On the other hand, Fig. 13a shows that the polyyne chains bridging between the SWNT base and the catalyst surface in the case of $\mathrm{Ni}_{38}$ were far greater in length. Generally, such polyyne chains were observed to be as large as $\mathrm{C}_{10}$ for $\mathrm{Ni}_{38}$ and $\mathrm{Ni}_{55}$ catalyst nanoparticles. In both Fe- and Ni-catalyst cases, continued SWNT growth was driven by the formation of polygonal carbon rings at the base of the SWNT, generally from the interaction of these bridging carbon chains. The length of these carbon chains therefore proved to be a critical factor in the context of the SWNT growth mechanism. For $\mathrm{Ni}_{38}$ and $\mathrm{Ni}_{55}$ catalysts, the rate of extension of these carbon chains was greater than the rate at which they self-isomerised, or 'collapsed' (Page et al., 2010a). In the case depicted in Fig. 13a, the extension and collapse of a single polyyne chain bound to the base of the growing $\mathrm{C}_{40}$ cap structure resulted in the formation of a conjugated 6-5-7-5 carbon ring system. Conversely, the rates of polyyne extension and collapse observed using $\mathrm{Fe}_{38}$ and $\mathrm{Fe}_{55}$ catalyst nanoparticles were generally more equivalent. SWNT growth was thus limited by the rate of polyyne chain extension. Ultimately these mechanistic differences yield Ni-catalysed SWNT growth rates ca. 69 $106 \%$ greater than those found using Fe-catalysed, for equivalent catalyst nanoparticle size. Somewhat unsurprisingly, the fundamental factor explaining the kinetic differences of Feand Ni-catalysed SWNT growth are the same as those which explain the differences in Feand Ni-catalysed SWNT nucleation. Fig. 12a and 13a show that, once again, the relative strengths of the Fe-C, Ni-C and C-C interactions correlate exactly with the observed SWNT nucleation kinetics. For example, the rate of SWNT growth is limited by the rate at which the bridging polyyne chains (pictured in Fig. 12a and 13a) can incorporate new carbon. This rate, in turn, is determined by the relative thermodynamics of $\mathrm{C}-\mathrm{C}$ bond formation in the presence of $\mathrm{Fe}$ and $\mathrm{Ni}$ atoms. As was discussed in \$3.3, the relative weakness of the Ni-C interaction means that, in a thermodynamic sense, the formation of $\mathrm{C}-\mathrm{C}$ bonds on $\mathrm{Ni}$ - 
catalysts is a more favorable process compared to that on Fe-catalysts. In this sense, therefore, the strength of the catalyst-carbon interaction constitutes a fundamental, guiding principle for understanding the mechanisms and kinetics of SWNT growth on different catalyst materials.

a

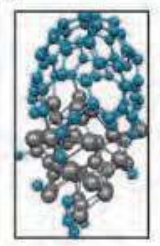

14.72 ps

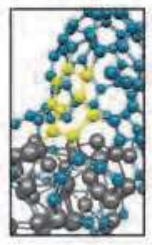

$33.08 \mathrm{ps}$

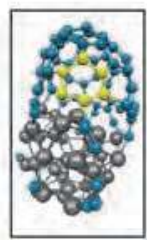

$17.06 \mathrm{ps}$

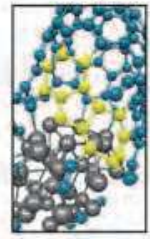

$33.38 \mathrm{ps}$

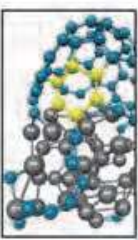

$17.68 \mathrm{ps}$

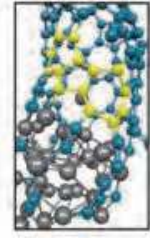

$42.82 \mathrm{ps}$

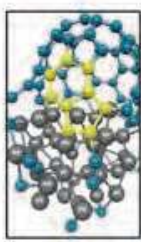

18.86 ps

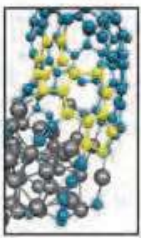

$42.94 \mathrm{ps}$

b

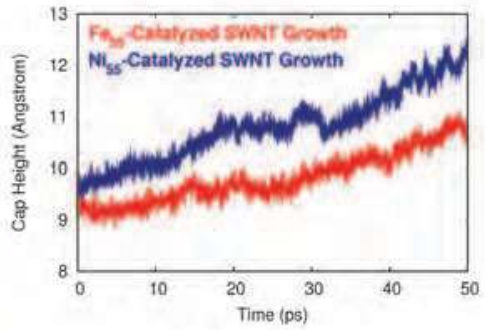

$(5,5) \mathrm{C}_{40} \mathrm{SWNT}$ cap on a $\mathrm{Ni}_{38}$ catalyst nanoparticle at $1500 \mathrm{~K}$. a) In this case, the extension and collapse of a single bridging polyyne chain results in the formation of an extended conjugated system at the base of the SWNT, including a hexagonal, heptagonal and two pentagonal carbon rings. Color conventions as in Fig. 6. b) Depending on the size of the catalyst nanoparticle, Ni-catalysed SWNT growth is found to be ca. $69-106 \%$ faster than Fe-catalysed SWNT growth at $1500 \mathrm{~K}$. (Adapted from (Page et al., 2010a). Reprinted with permission. (C) 2010 American Chemical Society)

\section{SWNT defects, healing and chirality-controlled growth}

As has been shown in $\$ 2-4$, there have been significant advances in both experimental and theoretical understanding of SWNT nucleation and growth on a number of different catalyst species. Yet there are still outstanding issues regarding phenomena associated with SWNT growth. The most notable phenomenon at present is that of 'chirality-controlled' growth. That is, a method by which a single particular $(n, m)$ chirality SWNT (or, at most a narrow distribution of $(n, m)$ SWNTs) may be synthesised in situ remains elusive to date. At the atomistic scale, chirality-controlled growth equates to growth in which only hexagonal rings are incorporated into the growth SWNT structure. The fundamental principles guiding such chirality-specific synthesis are, as yet, largely unknown. Such chirality-controlled growth is extremely desirable, since the physical, electrical and optical properties of a SWNT are determined entirely by its $(n, m)$ chiral indices. Current experimental SWNT synthesis techniques (such as CVD and arc-discharge) are known to produce a broad distribution of $(n, m)$ SWNTs. While it is possible to subsequently isolate a narrow distribution of $(n, m)$ SWNTs, such techniques invariably damage the SWNT structures by either chemical or physical means (Li et al., 2007; Zheng \& Semke, 2007). Such damage potentially limits the 
application of these SWNTs in nanoscale devices. An understanding of how to control a SWNTs chirality in situ is therefore critical in this respect.

\subsection{SWNT growth: An inherently defective process}

It was shown in $\$ 2-4$ that SWNT nucleation and growth are far from linear, ordered processes. On the contrary, they proceed via extremely complex pathways, resulting in disordered and unpredictable dynamics. Considering the temperature at which SWNTs nucleate and grow in CVD and arc-discharge environments (i.e. typically $1000 \mathrm{~K}$ or higher), this in itself is not so surprising. Yet it provides the greatest hurdle regarding the control of SWNT chirality, since these nonlinear dynamics result in the formation of a large number of defects in the SWNT structure during nucleation and growth. The high concentration of defect structures must be, to some extent, due to the various assumptions placed on these QM/MD simulations. Nevertheless, the formation of defect structures must also be attributable to the non-equilibrium conditions present during SWNT nucleation and growth. Indeed, by revisiting the discussion of $\$ 3.1-3.2$, it is apparent that the inclusion of defects (such as polyyne chains, non-hexagonal ring structures and vacancies in the $s p^{2}$-hybridised carbon network) is inherent to the SWNT nucleation process itself. The 'nucleus' of the SWNT itself on a transition metal catalyst is actually a pentagonal ring 'defect'. The subsequent ring condensation process, by which the SWNT cap fragment is formed, also produces a majority of non-hexagonal ring defects. Although this is attributed to the curvature of the catalyst surface imposing itself onto the growing $s p^{2}$-hybridised carbon network, the further formation of defect structures during growth (see §4) cannot be rationalized in this manner. Nevertheless, the incorporation of defect structures into a growing SWNT effectively alters its chirality, and therefore physical properties. Since it is apparent that SWNT nucleation and growth are inherently defect-inducing processes, it is important to understand the mechanisms by which such defects are removed in situ. $\mathrm{QM} / \mathrm{MD}$ simulation of such defect removal on transition metal catalysts is the subject of $\S 5.2$.

\subsection{SWNT healing: A fundamental aspect of chirality-controlled SWNT growth}

The removal of SWNT defect structures during growth has been investigated previously using QM/MD (Page et al., 2009). To induce growth gas-phase carbon atoms were adsorbed at the region between a model $\mathrm{C}_{40}$ cap and its supporting $\mathrm{Fe}_{38}$ catalyst surface. The hypothesis of this approach took into account the inherent stability of the C-C bond (relative to the Fe-C bond), and therefore the greater stability of the SWNT as a whole. Due to this stability, the removal, or healing, of defects during growth was anticipated to occur over longer time scales than those considered in prior QM/MD simulations (ca. $50 \mathrm{ps}$ ). Three gasphase carbon adsorption rates were therefore employed, viz. $1 \mathrm{C} / 0.5 \mathrm{ps} 1 \mathrm{C} / 10 \mathrm{ps}$ and $1 \mathrm{C}$ / 20 ps (rates denoted using 'fast', 'slow' and 'very slow'). It is noted here that the former of these adsorption rates is the same as that employed in the simulations discussed in $\S 4$.

Comparison of the three carbon adsorption rates is made in Fig. 14. It is immediate from this figure that the ability of the SWNT to heal itself during growth is directly correlated to the rate of carbon adsorption. As this rate decreases, the number of polygonal ring defects in the growing $s p^{2}$-hybridised carbon network decreases. Moreover, the active removal of defects from the growing SWNT structure, resulting in hexagonal ring formation is observed for slow and very slow carbon adsorption. This suggests that the kinetics of SWNT growth is more favorable compared to those of defect removal. Fig. 15 shows the reason explaining 
why this is indeed the case. This figure depicts schematically two examples of defect removal during slow SWNT growth. The first of these defects is a conjugated pentagonalheptagonal ring defect and the second is an adatom defect. In both cases, the defect is removed solely by the self-isomerization of the SWNT cap structure itself. For example, the adatom defect shown in Fig. $15 \mathrm{~b}$ is formed following the adsorption of a carbon atom onto an existing hexagonal ring at the SWNT base. This adatom defect quickly converted to a heptagonal ring defect, which is evidently significantly more stable (lasting for $c a .15 \mathrm{ps}$ ).

a

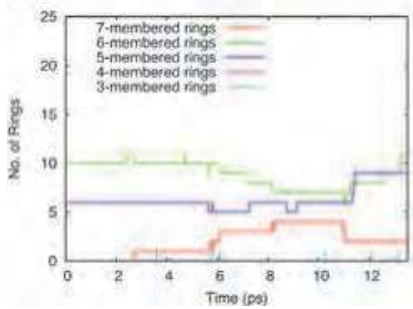

b

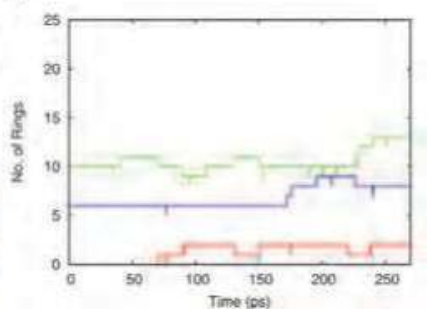

c

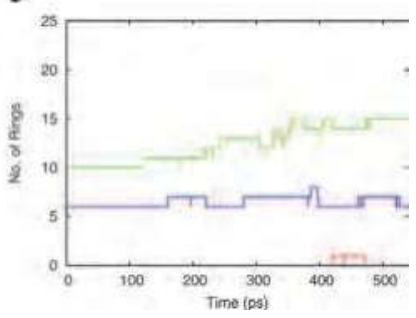

Fig. 14. SWNT healing is directly correlated with the rate of carbon adsorption at the SWNT base. Polygonal rings in formed a single SCC-DFTB/MD trajectory using adsorption rates of $1 \mathrm{C} / \mathrm{a}) 0.5 \mathrm{ps}, \mathrm{b}) 10 \mathrm{ps}$ and c) $20 \mathrm{ps}$. At the fastest adsorption rate considered, SWNT growth incorporates several defect ring structures into the SWNT structure. Slowing the adsorption rate to $1 \mathrm{C} / 10 \mathrm{ps}$, the incorporation of defects is suppressed due to the action of the self-isomerisation of the $s p^{2}$-hybridised carbon network. At the slowest rate considered, SWNT growth occurs solely due to hexagonal ring addition, thereby illustrating chiralitycontrolled SWNT growth. (Adapted from (Page et al., 2010c). Reprinted with permission. () 2011 American Chemical Society)

The addition of a second carbon atom results in a heptagonal-hexagonal ring rearrangement, which ultimately forms a $\mathrm{C}_{2}$ defect at the base of the SWNT. Following a further $c a .10 \mathrm{ps}$, this $\mathrm{C}_{2}$ unit detaches from the SWNT cap, and diffuses away over the catalyst surface. Both instances of SWNT defect removal depicted in Fig. 15 occur in the vicinity of the catalyst surface. The assistance of the catalyst surface is therefore implicated in these cases of SWNT healing. The timescales over which these two examples of self-isomerization take place are between 5 and 25 ps, respectively. This observation is indicative of a fundamental principle regarding the in situ control of SWNT chirality. That is, the rate at which defect structures are incorporated into the SWNT structure depends on the relative rates of defect addition (due to growth) and defect removal (due to SWNT self-isomerization).

\subsection{SWNT healing: Dependence on catalyst composition and size}

In $\$ 3.3$ and $\S 4.2$ it was established that a number of kinetic and mechanistic phenomena associated with SWNT nucleation and growth can be understood with recourse to the relative carbon-catalyst interaction strengths. For example, a stronger carbon-catalyst interaction leads to slower growth rates, and changes the mechanisms of SWNT nucleation and growth. From the previous section, it was seen that the catalyst nanoparticle is implicated in the SWNT healing process (\$5.2). It therefore seems reasonable to hypothesize that the carbon-catalyst interaction may also play some role regarding the relative ability of different catalysts to assist in SWNT healing processes. We will presently discuss such a proposal with respect to Fe and Ni-catalyst nanoparticles. 
a
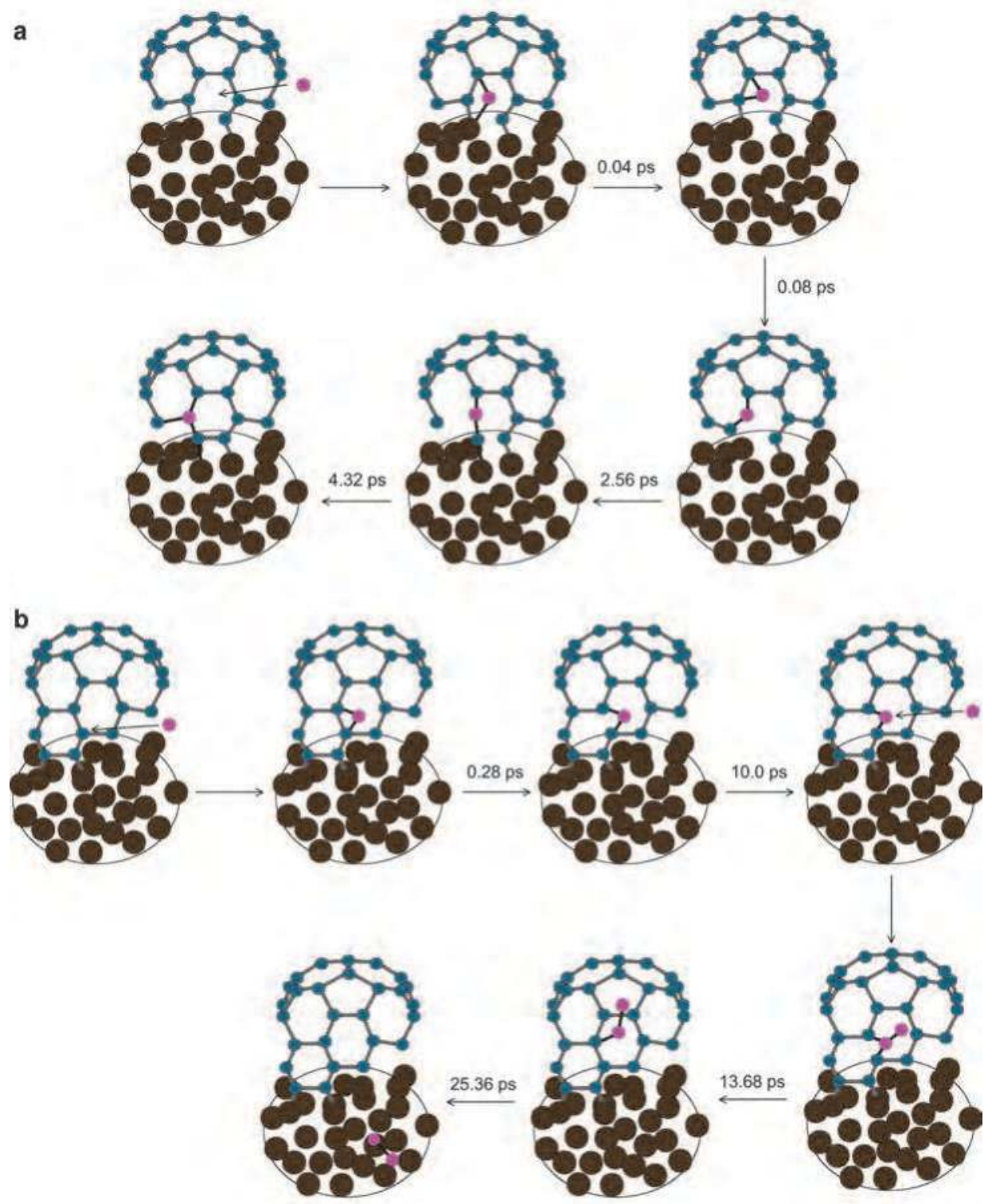

Fig. 15. Examples of SWNT healing observed during SWNT growth on $\mathrm{Fe}_{38}$ catalysts at 1500 K. In both cases, defects are removed from the growing SWNT cap solely by the selfisomerisation of the $s p^{2}$-hybridised carbon network. a) A conjugated pentagonal-heptagonal defect is removed, resulting in the formation of two hexagonal rings at the base of the SWNT cap. b) An adatom defect is removed, forming a hexagonal ring via a heptagonal ring defect intermediate structure. (Adapted from (Page et al., 2009). Reprinted with permission. (C) 2011 American Chemical Society) 


\begin{tabular}{|c|c|c|c|c|c|}
\hline & & $\mathrm{Fe}_{38}$ & $\mathrm{Ni}_{38}$ & $\mathrm{Fe}_{55}$ & $\mathrm{Ni}_{55}$ \\
\hline \multirow{6}{*}{ Defect Formation } & Pentagonal Formation & 3.2 & 6.1 & 2.2 & 4.6 \\
\hline & Heptagonal Formation & 0.2 & 0.4 & 0.3 & 0.2 \\
\hline & Hexagonal $\rightarrow$ Heptagonal Transformation & 2.7 & 2.8 & 2.3 & 2.9 \\
\hline & Hexagonal $\rightarrow$ Deformation & 1.0 & 0.2 & 0.6 & 0.2 \\
\hline & Hexagonal $\rightarrow$ Pentagonal Transformation & 0.1 & 0.3 & 0.6 & 0.1 \\
\hline & Total Defects Formed $\left(\Sigma_{1}\right)$ & 7.2 & 9.8 & 6.0 & 8.0 \\
\hline \multirow{4}{*}{ Defect Removal } & Hexagonal Formation & 3.4 & 3.3 & 3.4 & 2.9 \\
\hline & Heptagonal $\rightarrow$ Hexagonal Transformation & 1.1 & 1.0 & 0.8 & 1.3 \\
\hline & Pentagonal $\rightarrow$ Hexagonal Transformation & 1.2 & 1.7 & 1.5 & 1.6 \\
\hline & Total Defects Removed $\left(\Sigma_{2}\right)$ & 5.7 & 6.0 & 5.7 & 5.8 \\
\hline \multicolumn{2}{|l|}{ Net Healing $\left(\Sigma_{2}-\Sigma_{1}\right)$} & -1.5 & -3.8 & -0.3 & -2.2 \\
\hline
\end{tabular}

Table 1. SWNT healing statistics on Fe- and Ni-catalyst nanoparticles for a carbon adsorption rate of $1 \mathrm{C} / 10 \mathrm{ps}$. The net rate of SWNT healing may be considered as the difference between the rates of defect formation and defect removal. All data averaged over 10 SCC-DFTB/MD trajectories, following 300 ps of simulation.

QM/MD simulations of SWNT growth were carried out using $\mathrm{Fe}_{38}, \mathrm{Ni}_{38}, \mathrm{Fe}_{55}$ and $\mathrm{Ni}_{55}$ catalyst nanoparticles. Growth was induced at $1500 \mathrm{~K}$ using a slow carbon supply rate (i.e. 1 $\mathrm{C} / 10 \mathrm{ps}$ ). The average defect formation and defect removal statistics following $300 \mathrm{ps}$ are given in Table 1. For the purpose of this analysis, 'defect formation' is defined here as the formation of a new pentagonal or heptagonal ring, the conversion of a hexagonal ring to a pentagonal/heptagonal ring, or the destruction of a hexagonal ring (i.e. ring opening). Conversely, 'defect removal' is defined here as essentially the opposite of defect formation, i.e. the formation of new hexagonal rings and the conversion of pentagonal/heptagonal rings to hexagonal rings. From Table 1 it is evident that the rate of defect removal in the case of the four catalysts considered are essentially equivalent after 300 ps. Thus, there is little dependence of the defect removal process on the size, or elemental composition of the catalyst. This is reasonable, since the catalyst nanoparticle was never explicitly involved in the process of healing (as discussed in \$5.2). Rather, it plays an implicit role, by saturating dangling bonds at the edge of the SWNT structure, thereby supporting the selfisomerization process. On the other hand, Table 1 shows that the size, and more obviously, the elemental composition of the catalyst nanoparticle directly affects the rate of defect formation during growth. For example, for an equivalent catalyst size, SWNT growth on a Ni catalyst induces ca. 30-35\% more total defects, compared to SWNT growth on an Fe catalyst. For a particular type of metal, Table 1 also shows that the number of defects formed during SWNT growth decreases with increasing nanoparticle diameter. It is noted here that these two correlations are consistent with the effect of nanoparticle size and composition on the total SWNT growth rate, as discussed in §4.2. That is, faster growth leads to more defects, whereas slower growth leads to a smaller number of defects. 


\section{Conclusion}

We have reviewed our own recent investigations into the phenomena of SWNT nucleation and growth using state-of-the-art QM/MD methods. A summary of the primary conclusions discussed herein is provided in Fig. 16. The significance of the QM/MD method in this

a

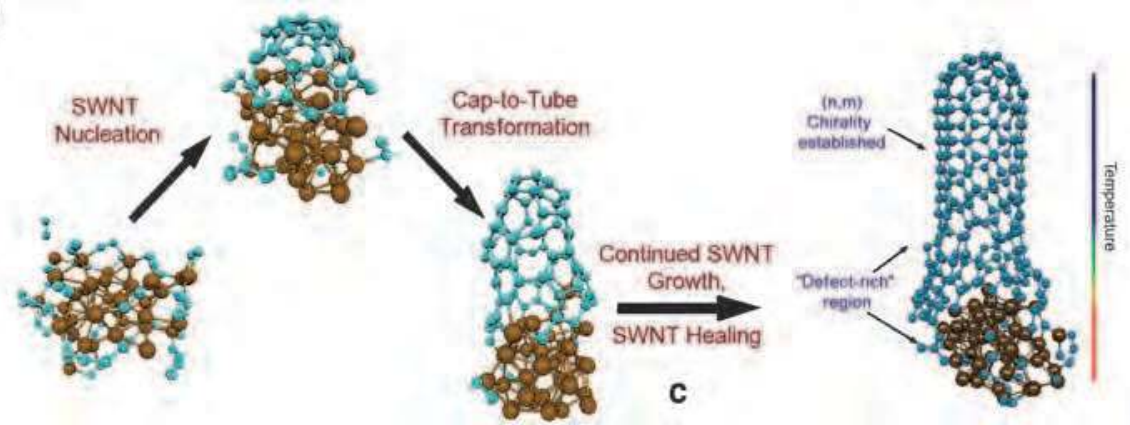

b

Adsorption of $\mathrm{CH}_{4}$ on $\mathrm{SiO}_{2}$

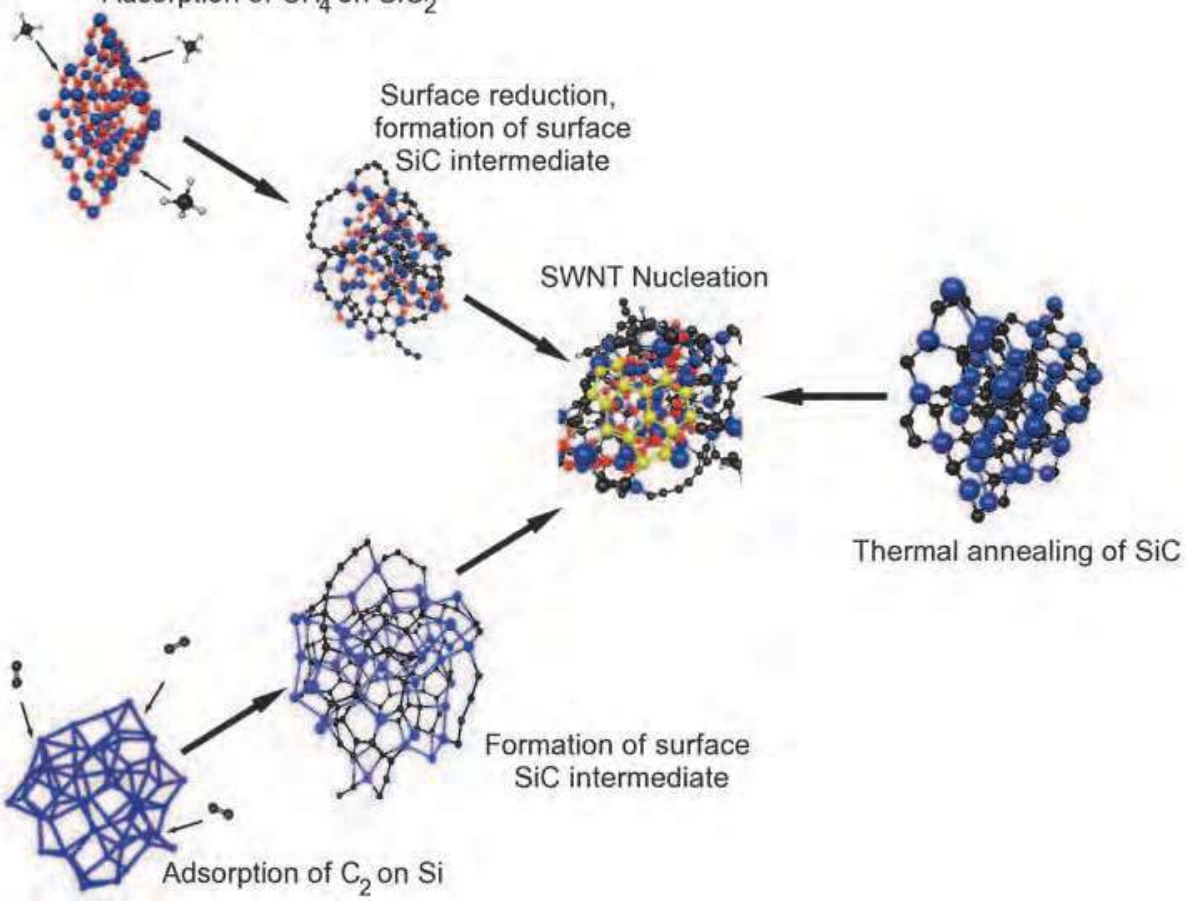

Fig. 16. Insights into the nucleation growth and defect-healing of SWNTs gained from QM/MD simulations. a) SWNT nucleation on transition metal nanoparticles begins with the oligomerisation of small carbon fragments on the catalyst surface. These units subsequently coalesce to form longer, extended polyyne chains which are able to isomerise/interact, thus forming polygonal carbon rings. SWNT nucleation and growth is then the result of 
continual polygonal ring condensation on the catalyst surface according to this mechanism. $\mathrm{QM} / \mathrm{MD}$ simulations also suggest that the removal of defect structures in the growing nanotube occurs via a natural process in which the isomerization of the SWNT $s p^{2}$ hybridised carbon network itself converts defects into hexagonal rings. This defect-healing occurs closest to the catalyst surface where the local temperature is hottest. As root growth continues, the established hexagonal rings in the cooler regions of the SWNT impart a templating effect on the healing process. Brown and cyan spheres represent Fe and $\mathrm{C}$ atoms, respectively. (Adapted from (Page et al., 2010c). Reprinted with permission. (c) 2011 American Chemical Society). b) SWNT nucleation on Si-based catalysts has been elucidated using QM/MD simulations. These simulations have established that a mechanism fundamentally different to that observed for transition metal catalysts is responsible for SWNT nucleation in this case. In particular, these catalyst nanoparticles remain in the solid phase throughout the nucleation process, and the saturation of the catalyst surface with carbon is a necessary prerequisite for SWNT nucleation. Accordingly, it is concluded that these cases of SWNT nucleation are explained with recourse to a VSS mechanism, as opposed to a VLS mechanism. QM/MD simulations have also established that this mechanism is independent of the catalyst employed, at least with respect to $\mathrm{SiO}_{2}, \mathrm{SiC}$ and $\mathrm{Si}$ catalysts. Blue, red and black spheres represent $\mathrm{Si}, \mathrm{O}$ and $\mathrm{C}$, respectively. Yellow spheres represent $\mathrm{C}$ atoms involved in SWNT nucleation.

context has therefore been demonstrated. QM/MD simulations of such non-equilibrium, high-temperature processes can provide fundamental knowledge that complements experimental understanding. Moreover, considering the spatial and temporal resolutions furnished by QM/MD methods (i.e. nanometers and picoseconds, respectively), and their physical reliability, such simulations can predate, or correct experimental understanding of these phenomena. This is certainly the case with respect to models of SWNT nucleation and growth. For example, the VLS mechanism of SWNT nucleation and growth on a variety of transition metal catalysts is very widely accepted. Yet it is only since the application of $\mathrm{QM} / \mathrm{MD}$ in this area that true understanding of various aspects of the VLS mechanism has come to light. One such aspect regards the atomistic processes of SWNT nucleation and growth, which are dominated by the formation and coalescence of extended polyyne chains, and the interaction of these chains with the supporting catalyst surface. Another aspect, which remains under debate at the time of writing, regards the existence and role of the transition metal carbide phase in the context of SWNT nucleation and growth. In particular, recent $\mathrm{QM} / \mathrm{MD}$ simulations and experiments have challenged the traditional role ascribed to this carbide phase in the SWNT nucleation process. QM/MD methods have also uncovered the phenomenon of defect healing during continued SWNT growth. This phenomenon, by which a SWNT structure consisting entirely of hexagons can be attained, has since been implicated in models of chirality-controlled growth. Most recently, QM/MD simulations have led the way in understanding the manner in which SWNTs nucleate and grow on 'non-traditional' catalysts including $\mathrm{SiO}_{2}, \mathrm{SiC}$ and $\mathrm{Si}$. They have also revealed the atomistic mechanism underpinning the CVD process on the former catalyst species. In this context, QM/MD methods alone have uncovered the remarkable fact that SWNT nucleation on these solid phase catalysts proceeds according to an entirely different mechanism, compared to the traditional picture of SWNT nucleation/growth on transition metal nanoparticles. Yet we do not acquiesce, and claim that QM/MD can offer nothing more in the understanding of SWNT nucleation and growth. There are still many aspects of these 
phenomena that remain misunderstood, and others that are currently under debate. The precise atomistic mechanism governing the termination of SWNT growth is once such aspect. Very recently it has been suggested (upon the basis of DFT calculations) that the phenomenon of Ostwald ripening plays an active role in the termination of SWNT growth (Börjesson \& Bolton, 2011). Nevertheless, it is clear that, as the computational technology continually advances, the understanding that can be gained from QM/MD simulations of such physical systems can only improve.

\section{Acknowledgement}

This work was in part supported by a CREST (Core Research for Evolutional Science and Technology) grant in the Area of High Performance Computing for Multiscale and Multiphysics Phenomena from the Japanese Science and Technology Agency (JST). Simulations were performed in part using the computer resources at the Research Centre for Computational Science (RCCS), Okazaki Research Facilities, National Institutes for Natural Sciences, and at the Academic Centre for Computing and Media Studies (ACCMS) at Kyoto University. A.J.P. acknowledges the Kyoto University Fukui Fellowship. S.I. acknowledges the Program for Improvement of Research Environment for Young Researchers from Special Coordination Funds for Promoting Science and Technology (SCF) commissioned by the Ministry of Education, Culture, Sports, Science and Technology (MEXT) of Japan for support.

\section{References}

Alder, B. J. \& Wainwright, T. E. (1957). Phase Transition for a Hard Sphere System. The Journal of Chemical Physics, Vol. 27, No. 5, pp. (1208-1209), 0021-9606

Amara, H., Bichara, C. \& Ducastelle, F. (2006). Formation of carbon nanostructures on nickel surfaces: A tight-binding grand canonical Monte Carlo study. Physical Review B, Vol. 73, No. 11, pp. (113404/1-113404/4), 1098-0121

Amara, H., Bichara, C. \& Ducastelle, F. (2008a). A Tight-Binding Grand Canonical Monte Carlo Study of the Catalytic Growth of Carbon Nanotubes. Journal of Nanoscience and Nanotechnology, Vol. 8, No. pp. (6099-6104), 1533-4880

Amara, H., Bichara, C. \& Ducastelle, F. (2008b). Understanding the Nucleation Mechanisms of Carbon Nanotubes in Catalytic Chemical Vapor Deposition. Physical Review Letters, Vol. 100, No. 5, pp. (056105/1-056105/4), 0031-9007

Amara, H., Roussel, J. M., Bichara, C., Gaspard, J. P. \& Ducastelle, F. (2009). Tight-binding potential for atomistic simulations of carbon interacting with transition metals: Application to the Ni-C system. Physical Review B, Vol. 79, No. 1, pp. (014109/1014109/17), 1098-0121

Andersen, H. C. (1980). Molecular dynamics simulations at constant pressure and/or temperature. The Journal of Chemical Physics, Vol. 72, No. 4, pp. (2384-2393), 00219606

Bachmatiuk, A., Börrnert, F., Grobosch, M., Schäffel, F., Wolff, U., Scott, A., Zaka, M., Warner, J. H., Klingeler, R., Knupfer, M., Büchner, B. \& Rümmeli, M. H. (2009). Investigating the Graphitization Mechanism of $\mathrm{SiO}_{2}$ Nanoparticles in Chemical Vapor Deposition. ACS Nano, Vol. 3, No. 12, pp. (4098-4104), 1936-0851 
Berendsen, H. J. C., Postma, J. P. M., van Gunsteren, W. F., DiNola, A. \& Haak, J. R. (1984). Molecular dynamics with coupling to an external bath. The Journal of Chemical Physics, Vol. 81, No. 8, pp. (3684-3690), 0021-9606

Börjesson, A. \& Bolton, K. (2011). First Principles Studies of the Effect of Ostwald Ripening on Carbon Nanotube Chirality Distributions. ACS Nano, Vol. 5, No. 2, pp. (771-779), 1936-0851

Brenner, D. W. (1990). Empirical Potential for Hydrocarbons for Use in Simulating the Chemical Vapor-Deposition of Diamond Films. Physical Review B, Vol. 42, No. 15, pp. (9458-9471), 0163-1829

Brenner, D. W. (1992). Empirical potential for hydrocarbons for use in simulating the chemical vapor deposition of diamond films. [Erratum to document cited in CA114(6):53045x]. Physical Review B, Vol. 46, No. pp. (1948), 0163-1829

Brenner, D. W., Shenderova, O. A., Harrison, J. A., Stuart, S. J., Ni, B. \& Sinnott, S. B. (2002). A second-generation reactive empirical bond order (REBO) potential energy expression for hydrocarbons. Journal of Physics: Condensed Matter, Vol. 14, No. pp. (783-802), 0953-8984

Cau, M., Dorval, N., Cao, B., Attal-Tretout, B., Cochon, J. L., Loiseau, A., Farhat, S. \& Scott, C. D. (2006). Spatial Evolutions of Co and Ni Atoms During SWNT Formation: Measurements and Modeling. Journal of Nanoscience and Nanotechnology, Vol. 6, No. 5, pp. (1298-1308), 1533-4880

Dai, H., Rinzler, A. G., Nikolaev, P., Thess, A., Colbert, D. T. \& Smalley, R. E. (1996). Singlewall nanotubes produced by metal-catalysed disproportionation of carbon monoxide. Chemical Physics Letters Vol. 260, No. pp. (471-475),

Ding, F., Bolton, K. \& Rosen, A. (2004a). Nucleation and growth of single-walled carbon nanotubes: A molecular dynamics study. Journal of Physical Chemistry B, Vol. 108, No. 45, pp. (17369-17377), 1520-6106

Ding, F., Bolton, K. \& Rosen, A. (2006a). Molecular dynamics study of SWNT growth on catalyst particles without temperature gradients. Computational Materials Science, Vol. 35, No. 3, pp. (243-246), 0927-0256

Ding, F., Rosen, A. \& Bolton, K. (2004b). Molecular dynamics study of the catalyst particle size dependence on carbon nanotube growth. The Journal of Chemical Physics, Vol. 121, No. 6, pp. (2775-2779), 0021-9606

Ding, F., Rosen, A. \& Bolton, K. (2004c). Size dependence of the coalescence and melting of iron clusters: A molecular-dynamics study. Physical Review B, Vol. 70, No. 7, pp. (075415/1-075415/6), 0163-1829

Ding, F., Rosen, A., Curtarolo, S. \& Bolton, K. (2006b). Modeling the melting of supported clusters. Applied Physics Letters, Vol. 88, No. pp. (133110/1-133110/3), 0003-6951

Dresselhaus, M. S., Dresselhaus, G. \& Avouris, P. (Ed.). (2001). Carbon Nanotubes: Synthesis, Structure, Properties and Applications, Springer-Verlag, 3-540-41086-4, Heidelberg

Dresselhaus, M. S., Dresselhaus, G. \& Eklund, P. C. (1996). Science of Fullerenes and Carbon Nanotubes, Academic Press, 978-0-12-221820-0, San Diego

Elstner, M., Porezag, D., Jungnickel, G., Elsner, J., Haugk, M., Frauenheim, T., Suhai, S. \& Seifert, G. (1998). Self-consistent-charge density-functional tight-binding method 
for simulations of complex materials properties. Physical Review B, Vol. 58, No. 11, pp. (7260-7268), 1098-0121

Eres, G., Rouleau, C. M., Yoon, M., Puretzky, A. A., Jackson, J. J. \& Geohegan, D. B. (2009). Model for Self-Assembly of Carbon Nanotubes from Acetylene Based on Real-Time Studies of Vertically Aligned Growth Kinetics. The Journal of Physical Chemistry C, Vol. 113, No. 35, pp. (15484-15491), 1932-7447

Esconjauregui, S., Whelan, C. M. \& Maex, K. (2009). The reasons why metals catalyze the nucleation and growth of carbon nanotubes and other carbon nanomorphologies. Carbon, Vol. 47, No. pp. (659-669),

Fan, X., Buczko, R., Puretzky, A. A., Geohegan, D. B., Howe, J. Y., Pantelides, S. T. \& Pennycook, S. J. (2003). Nucleation of Single-Walled Carbon Nanotubes. Physical Review Letters, Vol. 90, No. 14, pp. (145501/1-145501/4), 0031-9007

Farhat, S., de La Chapelle, M. L., Loiseau, A., Scott, C. D., Lefrant, S., Journet, C. \& Bernier, P. (2001). Diameter control of single-walled carbon nanotubes using argon-helium mixture gases. The Journal of Chemical Physics, Vol. 115, No. 14, pp. (6752-6759), 0021-9606

Futaba, D. N., Hata, K., Yamada, T., Mizuno, K., Yumura, M. \& Iijima, S. (2005). Kinetics of Water-Assisted Single-Walled Carbon Nanotube Synthesis Revealed by a TimeEvolution Analysis. Physical Review Letters, Vol. 95, No. 5, pp. (056104/1-4), 00319007

Gavillet, J., Loiseau, A., Journet, C., Willaime, F., Ducastelle, F. \& Charlier, J.-C. (2001). RootGrowth Mechanism for Single-Wall Carbon Nanotubes. Physical Review Letters, Vol. 87, No. 27, pp. (275504/1-275504/4), 0031-9007

Geohegan, D. B., A. A. Puretzky, D. Styers-Barnett, H. Hu, B. Zhao, H. Cui, C. M. Rouleau, G. Eres, J. J. Jackson, R. F. Wood, S. Pannala \& J. C. Wells. (2007). In situ timeresolved measurements of carbon nanotube and nanohorn growth. Physica Status Solidi (b), Vol. 244, No. 11, pp. (3944-3949), 1521-3951

Harris, P. J. F. (2007). Solid state growth mechanisms for carbon nanotubes. Carbon, Vol. 45, No. 2, pp. (229-239), 0008-6223

Harutyunyan, A. R., Awasthi, N., Jiang, A., Setyawan, W., Mora, E., Tokune, T., Bolton, K. \& Curtarolo, S. (2008). Reduced Carbon Solubility in Fe Nanoclusters and Implications for the Growth of Single-Walled Carbon Nanotubes. Physical Review Letters, Vol. 100, No. 19, pp. (195502/1-195502/4), 0031-9007

Heath, J., R. (1992). Synthesis of $\mathrm{C}_{60}$ from Small Carbon Clusters, In: Fullerenes: Synthesis, Properties and Chemistry of Large Carbon Clusters, G. S. Hammond and V. J. Kuck (Ed.), pp. (1-23), American Chemical Society, 0-8412-2182-0,

Homma, Y., Liu, H., Takagi, D. \& Kobayashi, Y. (2009). Single-walled carbon nanotube growth with non-iron-group "catalysts" by chemical vapor deposition. Nano Research, Vol. 2, No. 10, pp. (793-799), 1998-0124

Hoover, W. G. (1985). Canonical dynamics: Equilibrium phase-space distributions. Physical Review A, Vol. 31, No. 3, pp. (1695), 1050-2947

Huang, S., Cai, Q., Chen, J., Qian, Y. \& Zhang, L. (2009). Metal-Catalyst-Free Growth of Single-Walled Carbon Nanotubes on Substrates. Journal of the American Chemical Society, Vol. 131, No. 6, pp. (2094-2095), 0002-7863 
Huang, Z. P., Wang, D. Z., Wen, J. G., Sennett, M., Gibson, H. \& Ren, Z. F. (2002). Effect of nickel, iron and cobalt on growth of aligned carbon nanotubes. Applied Physics A: Materials Science \& Processing, Vol. 74, No. 3, pp. (387-391), 0947-8396

Iijima, S. (1991). Helical Microtubules of Graphitic Carbon. Nature, Vol. 354, No. 6348, pp. (56-58), 0028-0836

Iijima, S. \& Ichihashi, T. (1993). Single-Shell Carbon Nanotubes of 1-nm Diameter. Nature, Vol. 363, No. 6430, pp. (603-605), 0028-0836

Irle, S., Ohta, Y., Okamoto, Y., Page, A. J., Wang, Y. \& Morokuma, K. (2009). Milestones in molecular dynamics simulations of single-walled carbon nanotube formation: A brief critical review. Nano Research, Vol. 2, No. 10, pp. (755-767), 1998-0124

Irle, S., Zheng, G., Wang, Z. \& Morokuma, K. (2006). The $\mathrm{C}_{60}$ Formation Puzzle "Solved": QM/MD Simulations Reveal the Shrinking Hot Giant Road of the Dynamic Fullerene SelfAssembly Mechanism. Journal of Physical Chemistry B, Vol. 110, No. pp. (14531-14545), 1520-6106

Journet, C. \& Bernier, P. (1998). Production of carbon nanotubes. Applied Physics A: Materials Science E Processing, Vol. 67, No. 1, pp. (1-9), 0947-8396

Journet, C., Maser, W. K., Bernier, P., Loiseau, A., delaChapelle, M. L., Lefrant, S., Deniard, P., Lee, R. \& Fischer, J. E. (1997). Large-scale production of single-walled carbon nanotubes by the electric-arc technique. Nature, Vol. 388, No. 6644, pp. (756-758), 0028-0836

Krätschmer, W., Fostiropoulos, K. \& Huffman, D. R. (1990). The infrared and ultraviolet absorption spectra of laboratory-produced carbon dust: evidence for the presence of the $\mathrm{C}_{60}$ molecule. Chemical Physics Letters, Vol. 170, No. 2-3, pp. (167-170), 00092614

Kusunoki, M., Rokkaku, M. \& Suzuki, T. (1997). Epitaxial carbon nanotube film selforganized by sublimation decomposition of silicon carbide. Applied Physics Letters, Vol. 71, No. 18, pp. (2620-2622),

Li, X., Tu, X., Zaric, S., Welsher, K., Seo, W. S., Zhao, W. \& Dai, H. (2007). Selective Synthesis Combined with Chemical Separation of Single-Walled Carbon Nanotubes for Chirality Selection. Journal of the American Chemical Society, Vol. 129, No. 51, pp. (15770-15771), 0002-7863

Lindemann, F. A. (1910). The calculation of molecular vibration frequencies. Zeitschrift fur Physik, Vol. 11, No. pp. (609-612), 0340-2347

Liu, B., Ren, W., Gao, L., Li, S., Pei, S., Liu, C., Jiang, C. \& Cheng, H.-M. (2009a). MetalCatalyst-Free Growth of Single-Walled Carbon Nanotubes. Journal of the American Chemical Society, Vol. 131, No. 6, pp. (2082-2083), 0002-7863

Liu, B., Ren, W., Liu, C., Sun, C.-H., Gao, L., Li, S., Jiang, C. \& Cheng, H.-M. (2009b). Growth Velocity and Direct Length-Sorted Growth of Short Single-Walled Carbon Nanotubes by a Metal-Catalyst-Free Chemical Vapor Deposition Process. ACS Nano, Vol. 3, No. 11, pp. (3421-3430), 1936-0851

Liu, B., Tang, D.-M., Sun, C., Liu, C., Ren, W., Li, F., Yu, W.-J., Yin, L.-C., Zhang, L., Jiang, C. \& Cheng, H.-M. (2011). Importance of Oxygen in the Metal-Free Catalytic Growth of Single-Walled Carbon Nanotubes from $\mathrm{SiO}_{x}$ by a Vapor-Solid-Solid 
Mechanism. Journal of the American Chemical Society, Vol. 133, No. 2, pp. (197-199), 0002-7863

Liu, H., Takagi, D., Chiashi, S., Chokan, T. \& Homma, Y. (2010a). Investigation of Catalytic Properties of $\mathrm{Al}_{2} \mathrm{O}_{3}$ Particles in the Growth of Single-Walled Carbon Nanotubes. Journal of Nanoscience and Nanotechnology, Vol. 10, No. 6, pp. (4068-4073), 1533-4880

Liu, H., Takagi, D., Chiashi, S. \& Homma, Y. (2010b). The growth of single-walled carbon nanotubes on a silica substrate without using a metal catalyst. Carbon, Vol. 48, No. 1, pp. (114-122), 0008-6223

Liu, H., Takagi, D., Ohno, H., Chiashi, S., Chokan, T. \& Homma, Y. (2008). Growth of SingleWalled Carbon Nanotubes from Ceramic Particles by Alcohol Chemical Vapor Deposition. Applied Physics Express, Vol. 1, No. 1, pp. (014001/1-014001/3), 18820778

Martyna, G. J., Klein, M. L. \& Tuckerman, M. (1992). Nose-Hoover chains: The canonical ensemble via continuous dynamics. The Journal of Chemical Physics, Vol. 97, No. pp. (2635-2643), 0021-9606

Martyna, G. J., Tuckerman, M. E., Tobias, D. J. \& Klein, M. L. (1996). Explicit reversible integrators for extended systems dynamics. Molecular Physics: An International Journal at the Interface Between Chemistry and Physics, Vol. 87, No. 5, pp. (1117 - 1157), 0026-8976

Moisala, A., Nasibulin, A. G. \& Kauppinen, E. I. (2003). The role of metal nanoparticles in the catalytic production of single-walled carbon nanotubes - a review. Journal of Physics: Condensed Matter, Vol. 15, No. 42, pp. (S3011-S3035), 0953-8984

Mora, E. \& Harutyunyan, A. R. (2008). Study of Single-Walled Carbon Nanotubes Growth via the Catalyst Lifetime. Journal of Physical Chemistry C, Vol. 112, No. 13, pp. (48054812), 1932-7447

Neyts, E. C. \& Bogaerts, A. (2009). Numerical Study of the Size-Dependent Melting Mechanisms of Nickel Nanoclusters. Journal of Physical Chemistry C, Vol. 113, No. 7, pp. (2771-2776), 1932-7447

Nose, S. (1984). A unified formulation of the constant temperature molecular dynamics methods. The Journal of Chemical Physics, Vol. 81, No. 1, pp. (511-519), 0021-9606

Ohta, Y., Okamoto, Y., Irle, S. \& Morokuma, K. (2008). Rapid Growth of a Single-Walled Carbon Nanotube on an Iron Cluster: Density-Functional Tight-Binding Molecular Dynamics Simulations. ACS Nano, Vol. 2, No. 7, pp. (1437-1444), 1936-0851

Ohta, Y., Okamoto, Y., Page, A. J., Irle, S. \& Morokuma, K. (2009). Quantum Chemical Molecular Dynamics Simulation of Single-Walled Carbon Nanotube Cap Nucleation on an Iron Particle. ACS Nano, Vol. 3, No. 11, pp. (3413-3420), 1936-0851

Page, A. J., Chandrakumar, K. R. S., Irle, S. \& Morokuma, K. (2011a). Do $\mathrm{SiO}_{2}$ and CarbonDoped $\mathrm{SiO}_{2}$ Nanoparticles Melt? Insights from QM/MD Simulations and Ramifications Regarding Carbon Nanotube Growth. Chemical Physics Letters, In press

Page, A. J., Chandrakumar, K. R. S., Irle, S. \& Morokuma, K. (2011b). SWNT Nucleation from Carbon-Coated $\mathrm{SiO}_{2}$ Nanoparticles via a Vapor-Solid-Solid Mechanism. Journal of the American Chemical Society, Vol. 133, No. 3, pp. (621-628), 0002-7863 
Page, A. J., Irle, S. \& Morokuma, K. (2010a). Polyyne Chain Growth and Ring Collapse Drives Ni-Catalysed SWNT Growth: A QM/MD Investigation. Journal of Physical Chemistry C, Vol. 114, No. 18, pp. (8206-8211), 1932-7447

Page, A. J., Minami, S., Ohta, Y., Irle, S. \& Morokuma, K. (2010b). Comparison of singlewalled carbon nanotube growth from $\mathrm{Fe}$ and $\mathrm{Ni}$ nanoparticles using quantum chemical molecular dynamics methods. Carbon, Vol. 48, No. 11, pp. (3014-3026), 0008-6223

Page, A. J., Ohta, Y., Irle, S. \& Morokuma, K. (2010c). Mechanisms of Single-Walled Carbon Nanotube Nucleation, Growth, and Healing Determined Using QM/MD Methods. Accounts of Chemical Research, Vol. 43, No. 10, pp. (1375-1385), 0001-4842

Page, A. J., Ohta, Y., Okamoto, Y., Irle, S. \& Morokuma, K. (2009). Defect Healing during Single-Walled Carbon Nanotube Growth: A Density-Functional Tight-Binding Molecular Dynamics Investigation. Journal of Physical Chemistry C, Vol. 113, No. 47, pp. (20198-20207), 1932-7447

Page, A. J., Yamane, H., Ohta, Y., Irle, S. \& Morokuma, K. (2010d). QM/MD Simulation of SWNT Nucleation on Transition-Metal Carbide Nanoparticles. Journal of the American Chemical Society, Vol. 132, No. 44, pp. (15699-15707), 0002-7863

Porezag, D., Frauenheim, T., Kohler, T., Seifert, G. \& Kaschner, R. (1995). Construction of tight-binding-like potentials on the basis of density-functional theory: Application to carbon. Physical Review B, Vol. 51, No. 19, pp. (12947-12957), 1098-0121

Puretzky, A. A., Geohegan, D. B., Schittenhelm, H., Fan, X. \& Guillorn, M. A. (2002). Timeresolved diagnostics of single wall carbon nanotube synthesis by laser vaporization. Applied Surface Science, Vol. 197-198, No. 1, pp. (552-562), 0169-4332

Puri, P. \& Yang, V. (2007). Effect of Particle Size on Melting of Aluminum at Nano Scales. Journal of Physical Chemistry C, Vol. 111, No. 32, pp. (11776-11783), 1932-7447

Rahman, A. (1964). Correlations in the Motion of Atoms in Liquid Argon. Physical Review, Vol. 136, No. 2A, pp. (A405), 1050-2947

Raty, J. Y., Gygi, F. \& Galli, G. (2005). Growth of carbon nanotubes on metal nanoparticles: A microscopic mechanism from ab initio molecular dynamics simulations. Physical Review Letters, Vol. 95, No. 9, pp. (096103/1-096103/4), 0031-9007

Saito, Y. (1995). Nanoparticles and filled nanocapsules. Carbon, Vol. 33, No. 7, pp. (979-988), 0008-6223

Seifert, G., Porezag, D. \& Frauenheim, T. (1996). Calculations of molecules, clusters, and solids with a simplified LCAO-DFT-LDA scheme. International Journal of Quantum Chemistry, Vol. 58, No. 2, pp. (185-192), 1097-461X

Sharma, R., Rez, P., Treacy, M. M. J. \& Stuart, S. J. (2005). In situ observation of the growth mechanisms of carbon nanotubes under diverse reaction conditions. Journal of Electron Microscopy, Vol. 54, No. 3, pp. (231-237), 0022-0744

Shibuta, Y. \& Maruyama, S. (2002). Molecular dynamics simulation of generation process of SWNTs. Physica B, Vol. 323, No. pp. (187), 0921-4526

Shibuta, Y. \& Maruyama, S. (2003). Molecular dynamics simulation of formation process of single-walled carbon nanotubes by CCVD method. Chemical Physics Letters, Vol. 382, No. pp. (381-386), 0009-2614 
Shibuta, Y. \& Maruyama, S. (2007a). A molecular dynamics study of the effect of a substrate on catalytic metal clusters in nucleation process of single-walled carbon nanotubes. Chemical Physics Letters, Vol. 437, No. 4-6, pp. (218-223), 0009-2614

Shibuta, Y. \& Maruyama, S. (2007b). Bond-order potential for transition metal carbide cluster for the growth simulation of a single-walled carbon nanotube. Computational Materials Science, Vol. 39, No. pp. (842-848), 0927-0256

Sugai, T., Yoshida, H., Shimada, T., Okazaki, T., Shinohara, H. \& Bandow, S. (2003). New Synthesis of High-Quality Double-Walled Carbon Nanotubes by HighTemperature Pulsed Arc Discharge. Nano Letters, Vol. 3, No. 6, pp. (769-773), 15306984

Swope, W. C., Andersen, H. C., Berens, P. H. \& Wilson, K. R. (1982). A computer simulation method for the calculation of equilibrium constants for the formation of physical clusters of molecules: Application to small water clusters. The Journal of Chemical Physics, Vol. 76, No. 1, pp. (637-649), 0021-9606

Takagi, D., Hibino, H., Suzuki, S., Kobayashi, Y. \& Homma, Y. (2007). Carbon Nanotube Growth from Semiconductor Nanoparticles. Nano Letters, Vol. 7, No. 8, pp. (22722275), 1530-6984

Teo, K. B. K., Singh, C., Chhowalla, M. \& Milne, W. I. (2004). Catalytic Synthesis of Carbon Nanotubes and Nanofibers, In: Encyclopedia of Nanoscience and Nanotechnology, H. S. Nalwa (Ed.), pp. (665-686), American Scientific Publishers, 1-58883-001-2, Sevenson Ranch, California

Tersoff, J. (1988). New empirical approach for the structure and energy of covalent systems. Physical Review B, Vol. 37, No. 12, pp. (6991-7000), 0163-1829

Tersoff, J. (1989). Modeling solid-state chemistry: Interatomic potentials for multicomponent systems. Physical Review B, Vol. 39, No. 8, pp. (5566-5568), 0163-1829

Thess, A., Lee, R., Nikolaev, P., Dai, H. J., Petit, P., Robert, J., Xu, C. H., Lee, Y. H., Kim, S. G., Rinzler, A. G., Colbert, D. T., Scuseria, G. E., Tomanek, D., Fischer, J. E. \& Smalley, R. E. (1996). Crystalline ropes of metallic carbon nanotubes. Science, Vol. 273, No. 5274, pp. (483-487), 0036-8075

Wen, Y.-H., Zhang, Y., Zheng, J.-C., Zhu, Z.-Z. \& Sun, S.-G. (2009). Orientation-Dependent Structural Transition and Melting of Au Nanowires. Journal of Physical Chemistry C, Vol. 113, No. 48, pp. (20611-20617), 1932-7447

Woodcock, L. V. (1971). Isothermal molecular dynamics calculations for liquid salts. Chemical Physics Letters, Vol. 10, No. 3, pp. (257-261), 0009-2614

Xiang, R., Einarsson, E., Okawa, J., Miyauchi, Y. \& Maruyama, S. (2009). AcetyleneAccelerated Alcohol Catalytic Chemical Vapor Deposition Growth of Vertically Aligned Single-Walled Carbon Nanotubes. Journal of Physical Chemistry C, Vol. 113, No. 18, pp. (7511-7515), 1932-7447

Yao, Y., Liu, R., Zhang, J., Jiao, L. \& Liu, Z. (2007). Raman Spectral Measuring of the Growth Rate of Individual Single-Walled Carbon Nanotubes. Journal of Physical Chemistry C, Vol. 111, No. 24, pp. (8407-8409), 1932-7447

Yoshida, H., Shimizu, T., Uchiyama, T., Kohno, H., Homma, Y. \& Takeda, S. (2009). AtomicScale Analysis on the Role of Molybdenum in Iron-Catalysed Carbon Nanotube Growth. Nano Letters, Vol. 9, No. 11, pp. (3810-3815), 1530-6984 
Yoshida, H., Takeda, S., Uchiyama, T., Kohno, H. \& Homma, Y. (2008). Atomic-Scale In-situ Observation of Carbon Nanotube Growth from Solid State Iron Carbide Nanoparticles. Nano Letters, Vol. 8, No. 7, pp. (2082-2086), 1530-6984

Yoshinori, A. (2004). Carbon Nanotubes: Synthesis by Arc Discharge Technique, In: Encyclopedia of Nanoscience and Nanotechnology, H. S. Nalwa (Ed.), pp. (603-610), American Scientific Publishers, 1-58883-001-2, Sevenson Ranch, California

Zheng, G. S., Irle, S., Elstner, M. \& Morokuma, K. (2004). Quantum chemical molecular dynamics model study of fullerene formation from open-ended carbon nanotubes. Journal of Physical Chemistry A, Vol. 108, No. 15, pp. (3182-3194), 1089-5639

Zheng, M. \& Semke, E. D. (2007). Enrichment of Single Chirality Carbon Nanotubes. Journal of the American Chemical Society, Vol. 129, No. 19, pp. (6084-6085), 0002-7863 


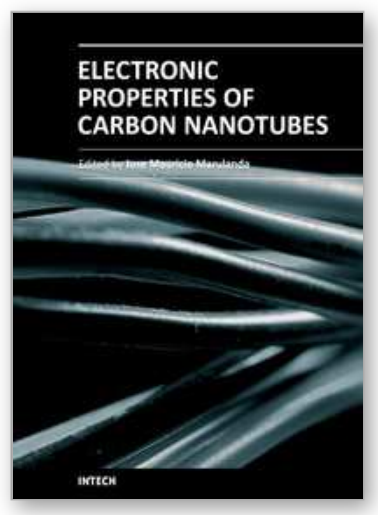

\author{
Electronic Properties of Carbon Nanotubes \\ Edited by Prof. Jose Mauricio Marulanda
}

ISBN 978-953-307-499-3

Hard cover, 680 pages

Publisher InTech

Published online 27, July, 2011

Published in print edition July, 2011

Carbon nanotubes (CNTs), discovered in 1991, have been a subject of intensive research for a wide range of applications. These one-dimensional (1D) graphene sheets rolled into a tubular form have been the target of many researchers around the world. This book concentrates on the semiconductor physics of carbon nanotubes, it brings unique insight into the phenomena encountered in the electronic structure when operating with carbon nanotubes. This book also presents to reader useful information on the fabrication and applications of these outstanding materials. The main objective of this book is to give in-depth understanding of the physics and electronic structure of carbon nanotubes. Readers of this book should have a strong background on physical electronics and semiconductor device physics. This book first discusses fabrication techniques followed by an analysis on the physical properties of carbon nanotubes, including density of states and electronic structures. Ultimately, the book pursues a significant amount of work in the industry applications of carbon nanotubes.

\title{
How to reference
}

In order to correctly reference this scholarly work, feel free to copy and paste the following:

Alister J. Page, Krs Chandrakumar, Ying Wang, Stephan Irle and Keiji Morokuma (2011). Mechanisms of Single-Walled Carbon Nanotube Nucleation, Growth and Chirality-Control: Insights from QM/MD Simulations, Electronic Properties of Carbon Nanotubes, Prof. Jose Mauricio Marulanda (Ed.), ISBN: 978-953-307-499-3, InTech, Available from: http://www.intechopen.com/books/electronic-properties-of-carbonnanotubes/mechanisms-of-single-walled-carbon-nanotube-nucleation-growth-and-chirality-control-insightsfrom-qm

\section{INTECH}

open science | open minds

\section{InTech Europe}

University Campus STeP Ri

Slavka Krautzeka 83/A

51000 Rijeka, Croatia

Phone: +385 (51) 770447

Fax: +385 (51) 686166

www.intechopen.com

\section{InTech China}

Unit 405, Office Block, Hotel Equatorial Shanghai

No.65, Yan An Road (West), Shanghai, 200040, China 中国上海市延安西路65号上海国际贵都大饭店办公楼 405 单元

Phone: +86-21-62489820

Fax: $+86-21-62489821$ 
(C) 2011 The Author(s). Licensee IntechOpen. This chapter is distributed under the terms of the Creative Commons Attribution-NonCommercialShareAlike-3.0 License, which permits use, distribution and reproduction for non-commercial purposes, provided the original is properly cited and derivative works building on this content are distributed under the same license. 